\title{
VIOLA PYRENAICA RAMOND EX DC IN THE NORTHERN PART OF THE DINARIC MOUNTAINS (THE PLATEAUS OF TRNOVSKI GOZD AND NANOS, SLOVENIA)
}

\author{
Igor DAKSKOBLER* \& Jernej PELJHAN**
}

\begin{abstract}
The article describes new localities of a south-European montane species Viola pyrenaica in the Julian Alps and in the Trnovski gozd and Nanos plateaus (the northern part of the Dinaric mountains). It has been established that in the localities known so far in Slovenia, the species grows on similar sites as elsewhere in the Alps and other mountain ranges of the Balkan Peninsula, above all on overgrown screes, stony grasslands, on forest edges, in light forests on stony ground and in tall herb communities on calcareous bedrock in the submontane, montane and subalpine belt (450 to $1600 \mathrm{~m}$ a.s.l.). The floristic composition of the communities in which it grows is presented in four tables. On the Trnovski gozd plateau it was found in various successional stages of abandoned pastures or grasslands of the association Genisto sericeae-Seslerietum kalnikensis and in open coppice stands of hop hornbeam and flowering ash, which are classified into the association Seslerio autumnalis-Ostryetum carpinifoliae.

Key words: Viola pyrenaica, syntaxonomy, Carpinion orientalis, Satureion subspicatae, the Julian Alps, the Trnovski gozd plateau, the Nanos plateau, the Dinaric mountains, Slovenia.

\section{Izvleček}

V članku smo opisali nova nahajališča južnoevropske montanske vrste Viola pyrenaica v Julijskih Alpah ter v Trnovskem gozdu in pod Nanosom (severni del Dinarskega gorstva). Ugotavljamo, da ta vrsta na doslej znanih nahajališčih v Sloveniji uspeva na podobnih rastiščih kot drugod v Alpah in v gorovjih Balkanskega polotoka, predvsem na poraslem grušču, v kamnitih traviščih, na gozdnih robovih, v svetlih kamnitih gozdovih in $\mathrm{v}$ združbah visokih steblik na apnenčasti podlagi v submontanskem, montanskem in subalpinskem pasu (450 do $1600 \mathrm{~m}$ nm. v.). Floristično sestavo združb, v katerih uspeva, prikazujemo v štirih tabelah. V Trnovskem gozdu smo jo našli v različnih stadijih zaraščanja na traviščih iz asociacije Genisto sericeae-Seslerietum kalnikensis in v vrzelastih panjevskih sestojih črnega gabra in malega jesena, ki jih uvrščamo v asociacijo Seslerio autumnalisOstryetum carpinifoliae.
\end{abstract}

Ključne besede: Viola pyrenaica, sintaksonomija, Carpinion orientalis, Satureion subspicatae, Julijske Alpe, Trnovski gozd, Nanos, Dinarsko gorstvo, Slovenija.

\section{INTRODUCTION}

In Slovenia, the south-European montane species Viola pyrenaica has had the status of rare species so far and is included into the Red list of vascular plants (Wraber \& Skoberne 1989: 336, Wraber et al. 2002: 8910). During our research in the last few years (Surina 2004: 232-233, Dakskobler 2005: 183-184) we have discovered several new localities and concluded that on account of its early blooming the species has probably been a slightly overlooked representative of Slovenian montane (Alpine) flora. This hypothesis was tested in 2006 and the results are presented in this article.

\footnotetext{
* Institute of Biology, Scientific Research Centre of the Slovenian Academy of Sciences and Arts, Regional unit Tolmin, Brunov drevored 13, SI - 5220 Tolmin. Igor.Dakskobler@guest.arnes.si

** Col 68 a, SI-5273 Col, Slovenia. Jernejpeljhan@gmail.com
} 


\section{METHODS}

The flora and vegetation were researched applying the standard Central-European methods (Ehrendorfer \& Hamman 1965, Braun-Blanquet 1964). Field data (floristic and phytosociological relevés) were entered into the FloVegSi database (T. Seliškar et al. 2003). This application was used also in the preparation of maps, including the distribution map. The relevés were arranged into the phytosociological tables applying numerical methods (hierarchical classification, PCoA ordination method). Program package SYN-TAX (Podani 2001) was used. Combined cover-abundance values were transformed into the ordinal scale following van der Maarel (1979). The nomenclature follows the Mala flora Slovenije (Martinčič et al. 1999) for vascular plants, and Frahm \& Frey (1992) and Martinčič (2003) for mosses. Syntaxonomical units and their authors are listed in the Appendix. When describing new localities we used basic topographical maps of the Republic of Slovenia 1:5000 and 1: 10000 (GURS), as well as the Atlas of Slovenia in the scale of 1: 50000 (3rd edition, 1996).

\section{RESULTS}

\subsection{Distribution of Viola pyrenaica in the Julian Alps}

Viola pyrenaica is disjunctly distributed in the larger part of the Alps as well as in other south- and Central-European mountain ranges (Aeschimann et al. 2004: 436). The localities known so far in the Julian Alps (Mayer 1954: 10, Wraber \& Skoberne 1989: 336, Surina 2004: 232-233, Dakskobler 2005: 183-184) are now supplemented with the following new data (written in bold are the localities in the new quadrants of the Central-European flora mapping):

9749/4 (UTM VM11): the Bača Valley, Trtnik, around 630 to $700 \mathrm{~m}$ a.s.l., road bank, breccia, stony grassland. Leg. \& det. I. Dakskobler, 1. 4. 2006 and 17. 3. 2007, working herbarium ZRC SAZU.

9749/4 (UTM VM12): the Bohinj region, Lisec near Mt. Črna prst, the saddle under Mali Lisec, $1590 \mathrm{~m}$ a.s.l., in a tall herb community (Centaureo julicae-Laserpitietum sileris s. lat. - relevé 1 in Table 1). Leg. \& det. I. Dakskobler, 9. 8. 2006, working herbarium ZRC SAZU.
9749/4 (UTM VM22): the Bača Valley, stony gullies, stony grasslands, tall herb communities under the Slatnik ridge, at about 1250 to $1420 \mathrm{~m}$ a.s.l. Leg. \& det. I. Dakskobler, 14. 5. 2006, working herbarium ZRC SAZU.

9749/1 (UTM VM12): the Bohinj region, the left bank of the Sava Bohinjka river, at about $520 \mathrm{~m} \mathrm{~s}$. s. 1., screes under Rsnik, which are overgrown with pioneer woods of Picea abies, Fraxinus excelsior, Acer pseudoplatanus, Carpinus betulus, Tilia platyphyllos, Corylus avellana, Frangula alnus etc. Leg. \& det. I. Dakskobler, I. Veber \& B. Zupan, 25. 5. 2007, working herbarium ZRC SAZU.

9750/3 (UTM VM22): the Bača Valley, stony grasslands and gullies on the sunny slopes of the Lajnar ridge above the upper Bača Valley, near Kovce, at about 1200 to $1350 \mathrm{~m}$ a.s.l. Leg. \& det. I. Dakskobler, 14. 5. 2006, working herbarium ZRC SAZU.

9747/1 (UTM UM82): the Krn mountains, the slopes of Morizna opposite the village of Trnovo: Labrje, at about 490 to $660 \mathrm{~m}$ a.s.l., limestone, hillside scree (talus), debris rocks, a hop hornbeam and flowering ash community (Fraxino orni-Ostryetum s. lat.) - relevés 1 to 5 in Table 3. Leg. \& det. I. Dakskobler, 12. 5. 2006, working herbarium ZRC SAZU.

9647/4 (UTM UM93): the Bovec region, the Lepena valley, the left bank of the Lepenica along the snowslip under Peščenek: under Bulovec, stony grassland, together with Genista radiata, Festuca calva, Primula veris subsp. columnae, Vinca minor, Verbascum lychnitis, Carduus crassifolius, Aconitum angustifolium, A. lycoctonum, Fragaria vesca, Cruciata glabra, Viola riviniana, Dactylis glomerata, Mercurialis perennis, Calamagrostis varia etc., at about $460 \mathrm{~m}$ a.s.l. Leg. \& det. I. Dakskobler, 4. 5. 2006, working herbarium ZRC SAZU.

9647/4 (UTM UM92): the Bovec region, the Lepena valley, on the right bank of the Lepenica: stony grassland opposite the Kavšč homestead, at about $480 \mathrm{~m}$ a.s.l., together with Brachypodium rupestre, Bromus erectus agg., Cytisus pseudoprocumbens, Chamaecytisus purpureus, Medicago falcata, Acinos alpinus etc. Leg. \& det. I. Dakskobler, 16. 5. 2006, working herbarium ZRC SAZU.

9648/3 (UTM UM92): the Bovec region, the upper part of the Lepena valley, stony meadows and for- 
est edges along the road towards the end of the valley near the Štefan homestead and upstream from the Gajger homestead at about 620 to $670 \mathrm{~m}$ a.s.l.; stony grasslands under Okroglica near the path to the Lepenica waterfall, at about $800 \mathrm{~m}$ a.s.l. Leg. \& det. I. Dakskobler, 4. 5. 2006, working herbarium ZRC SAZU.

9648/4 (UTM VM02): the Bohinj region, hillside scree under Stador, above the Črno jezero, in open pioneer stand of Acer pseudoplatanus, $1350 \mathrm{~m}$ a.s.l. Leg \& det. I. Dakskobler \& B. Zupan, 7. 9. 2007, working herbarium ZRC SAZU.

9647/1 (UTM UM83): the Bovec region, the Možnica valley, the upper part of the valley between Mirnik and Korita, hillside scree under the Jezersko sedlo-Lašte ridge (to the west of Mt. Jerebica), at about 930 to $1050 \mathrm{~m}$ a.s.l., communities on overgrown screes - see Table 2. Leg. \& det. I. Dakskobler, 26. 6. 2006, working herbarium ZRC SAZU.

9646/4 (UTM UM72): The Bovec region, the Učja valley, on the slopes of Mt. Vrh Planje, on localities Pod Pustim gozdom and Pusti gozd, at about 750 to $950 \mathrm{~m}$ a.s. 1., dolomite limestone, rendzina, pioneer woods (Ostrya carpinifolia, Fraxinus ornus, Fraxinus excelsior) on abandoned formerly hay-fields and pastures, partly in beech forest (Ostryo-Fagetum). Leg. \& det. I. Dakskobler, 23. 4. 2007, working herbarium ZRC SAZU (in the same area also in Italian territory, at about $1140 \mathrm{~m}$ a.s.l, on stony grassland with dominant Genista radiata and Festuca calva).

\subsection{Characterization of the Viola pyrenaica sites in the Julian Alps}

In the Julian Alps, Viola pyrenaica has so far been recorded in the altitudinal belt at between 450 and $1600 \mathrm{~m}$ a.s.l., i.e. in the submontane, montane, altimontane and subalpine belt, up to, or just above, the timberline. Geological bedrock is limestone and (or), dolomite, but also common is hillside scree and (or) debris rocks. The soil is shallow, raw (Lithosols) or rendzina. The species was recorded in a wet rock crevice community together with Saxifraga hostii (the locality Home near Mt. Črna prst), on moist, slightly nitrophilous and already quite overgrown montane screes (the Možnica valley, Table 2), on subalpine screes which are being overgrown with shrub or tree species - Rhamnus fal- lax, Rubus idaeus, Acer pseudoplatanus (the locality Home near Mt. Črna prst, under Stador above the Črno jezero), on stony dealpine grasslands from the class Festuco-Brometea (the Lepena valley opposite the Kavšč homestead, Trtnik near Podbrdo), in tall herb communities (associations Centaureo julicae-Laserpitietum sileris - Table 1, Eryngio alpinae-Caricetum ferrugineae - Dakskobler et al. 2005, Table 1) - under Mts. Šavnik, Lajnar, Kobla and Lisec and in Home, on stony subalpine grasslands with Genista radiata (under Planina nad Sočo and under Vrh Planje in the Učja valley), on subalpine grasslands with Festuca calva as the dominant species - Avenastro parlatorei-Festucetum calvae (under Škrbina in the Krn mountains - Surina 2004), in stony hop hornbeam and flowering ash coppice forests (Fraxino orni-Ostryetum s. lat., inc. Cytisantho-Ostryetum, Seslerio albicantis-Ostryetum): Podčela near Bovec - relevés 6-8 in Table 3, Morizna - relevés 1 to 5 in Table 3, Izgora, also in stony pioneer hop hornbeam forests on beech sites (relevé 9 in Table 3) and in beech forests (Ostryo-Fagetum, Ranunculo platanifolii-Fagetum) - the Bala and the Učja valleys. Sunny aspects prevail, although it inhabits also shady aspects (e.g. in the Lepena valley). In the Julian Alps it has been recorded in communities of the following classes, orders and alliances: Elyno-Seslerietea (alliance Caricion austroalpinae); Festuco-Brometea (alliance Mesobromion = Bromion erecti), Querco-Fagetea $($ order Quercetalia pubescentis, alliance Carpinion orientalis; order Fagetalia sylvaticae, alliance Aremonio-Fagion), EricoPinetea (alliance Erico-Fraxinion orni); its occurrence was determined also in transitional communities, successional stages which are similar to the communities of classes Mulgedio-Aconitetea (order Adenostyletalia), Thlaspietea rotundifolii (order Stipetalia calamagrostis) and Trifolio-Geranietea (order Origanetalia vulgaris). Since it occurs in various communities, it is difficult to assess its phytosociological affinity or characterization as a diagnostic species of some of the above-mentioned higher syntaxonomic units. Aeschimann et al. (2004: 436) characterize it with a questionmark (?) as a diagnostic species of subalpine spruce forests, rich in tall herbs (suballiance Chrysanthemo rotundifoliae-Piceenion).

\subsection{Distribution of Viola pyreniaca in the southeastern European mountains}

In general, Viola pyrenaica Ramond ex DC $(=V$. sciaphila Koch) is distributed in south- European mountains from Cantabria and the Pyrenees to 
the Caucasus (Gams \& Becker 1925: 645, Strid 1989: 616, Aichele \& Schwegler 1995: 277). According to Valentine et al. (1968: 273) it thrives on subalpine sites in the Pyrenees, the Alps, in the Central Apennines and in the mountain ranges of the Balkan Peninsula. Hess et al. (1977: 740) mention similar distribution. Meusel et al. (1978: 170) characterize it as (Rifanian) + Pyrenean + Cottian- northern- and eastern-Alpine (disjunct)Illyrian-eastern-Mediterranean montane floral element. The locality in the Central Apennines is mentioned also by Pignatti (1982: 106). Hayek (1927, reprint from 1975: 504) describes localities in the Balkans, namely in Serbia, Bulgaria and Greece (Tessalia, Epirus). Its sites and distribution in Greece are described also by Strid (1989: 616). According to Hayek (ibid.), the taxon Viola prenja Beck (= V. pyrenaica var. prenja K. Maly) grows in Bosnia and Herzegovina. In Albania, Viola pyrenaica grows in the subalpine belt (Qosja et al. 1992: 304). Trinajstić (1975: 46-47) reports that within former Yugoslavia the species was distributed in Slovenia, Bosnia and Herzegovina and in Serbia, inhabiting dry grasslands, subalpine pastures and rocks (rockwalls). He associates Viola pyrenaica with the taxon $V$. prenja $=V$. pyrenaica var. prenja. Diklić (1972: 142) lists its localities in eastern (Rtanj) and southeastern Serbia (Sarlak). Here, this species grows on shady rockwalls, on scree slopes, on subalpine meadows, in light montane and subalpine forests and scrub communities, mostly (although not exclusively) on limestone. Similar stony sites are inhabited by Viola pyrenaica also in territory of the Republic of Macedonia, in mountain chains of Galičica and Bistra (Erben 1995: 514). Željka Bjelčić (in litt.) forwarded the list of its localities in Bosnia and Herzegovina, which is followed also in this article. The herbarium of the National museum (Zemaljski muzej) in Sarajevo (SARA) keeps specimens of this species, collected by K. Maly in Mt. Preslica near Ivan planina (1300 m a.s.1.), in Mt. Palež near Trebević planina (770 to $840 \mathrm{~m}$ a.s.l.) and on Jahorina (Kalaidžin Kamen,1420 m a.s.l.). Brandis collected the species near Travnik (Derventa). Beck (1916: 367 or 1919: 191) reports that Pyrenean violet in Bosnia and Herzegovina grows on cold habitats from the montane to the alpine belt. He mentions a localities in Bosnia (Gajić, Donje Hrasno). Surina (in litt.) recorded the violet on Troglav above Livanjsko polje, in the stand of the association Festucetum bosniacae at the altitude of about $1700 \mathrm{~m}$. So far, we have not had information on the growth of Viola pyrenaica in the north- ern part of the Dinaric mountains in Slovenia nor in Croatia (Šegulja 1997: 17-18, Nikolić, in litt.)

\subsection{Localities and sites of Viola pyrenaica on the southern edge of the Trnovski gozd plateau and under the Nanos plateau (the northern part of the Dinaric mountains)}

0149/2 (UTM VL18): the southern edge of the Trnovski gozd plateau between Col and Otlica, near the village of Gozd, stony sites on and under the Sončnica - Podrta gora ridge and onward towards Leskov školj; limestone, 870 to $900 \mathrm{~m}$ a.s.l. (Figure 2 and Table 4). Leg. \& det. I. Dakskobler \& J. Peljhan, 27. 9. 2005, J. Peljhan, 9. 4. 2006 and I. Dakskobler, 21. 4. 2006, working herbarium ZRC SAZU.

0150/3 (UTM VL27): the slopes of the Nanos plateau above the village of Podnanos, near the road Podnanos-Nanos, limestone, stony sites on forest edge between coppice forest of Ostrya carpinifolia and Quercus pubescens and steep rocky slopes, 550 m a.s.l. Leg. \& det. I. Dakskobler \& B. Vreš, 24. 5. 2007, working herbarium ZRC SAZU.

Viola pyrenaica was found on the southern edge of the Trnovski gozd plateau, on the ridge between Col and Otlica, at the contact of the Dinaric and sub-Mediterranean phytogeographical region of Slovenia (according to Wraber 1969) - Figures 1, 2. It grows on overgrown rocky ground (relevés 1 and 2 in Table 4), on forest edges (relevé 6 in Table 4 ), on steep stony slopes, in gullies and on former pastures that are being overgrown with tall herbs. These are mostly various stages of overgrowth of stony grasslands from the association Genisto sericeae-Seslerietum kalnikensis (relevés 1 to 9 in Table 4), which have not yet been given a more thorough synsystematic classification. Often they are the stages with Laserpitium siler as their dominant species.

Viola pyrenaica was recorded also in light coppice forest stands, under the ridge, on sunny slopes above the Vipava Valley (relevés 10 to 13 in Table 4). These stands, dominated by hop hornbeam (Ostrya carpinifolia) and flowering ash (Fraxinus ornus), with addition of whitebeam (Sorbus aria), pubescent oak (Quercus pubescens) and in places also beech (Fagus sylvatica) and Montpellier maple 


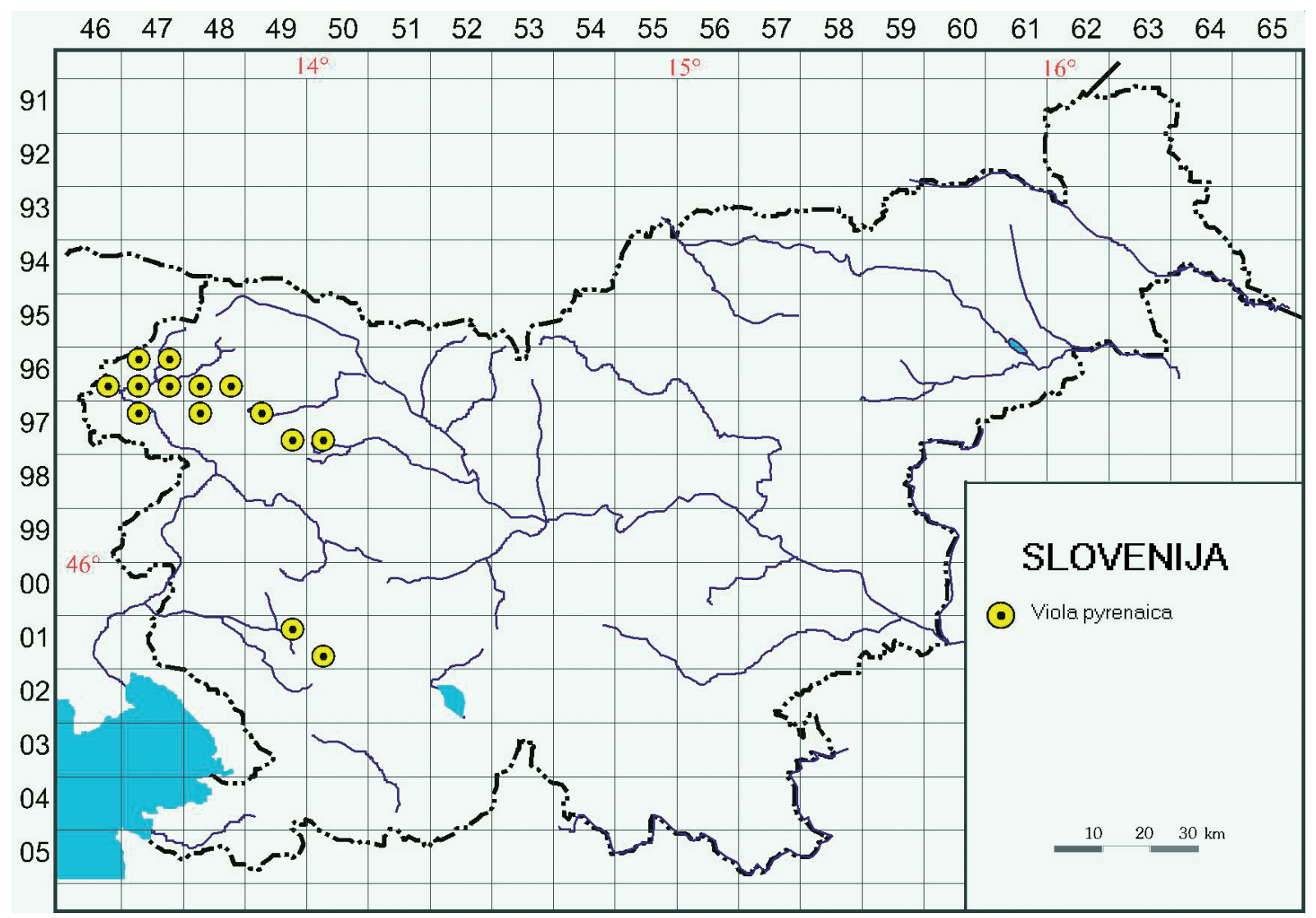

Figure 1: Distribution of Viola pyrenaica in Slovenia / Slika 1: Razširjenost vrste Viola pyrenaica v Sloveniji

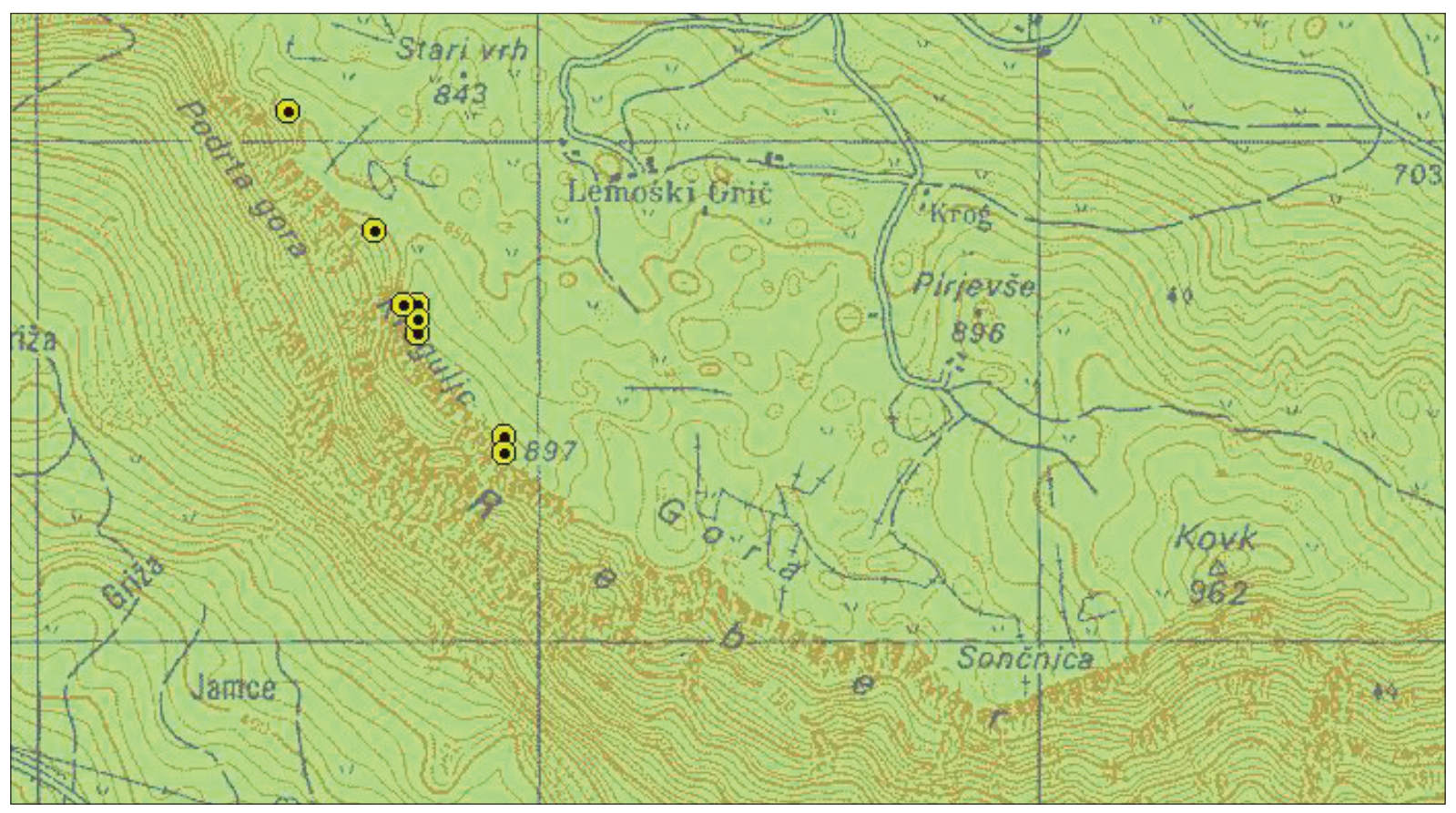

Figure 2: Localities of Viola pyrenaica in the southern part of the Trnovski gozd plateau (Source: State topographical map 1: 25 000, GURS)

Slika 2: Nahajališča vrste Viola pyrenaica na južnem robu Trnovskega gozda (Vir: Državna topografska karta RS 1: 25 000, GURS) 
(Acer monspessulanum), are classified into the association Seslerio autumnalis-Ostryetum carpinifoliae. These, at least in part, secondary stands originated on potential sites of beech communities from the associations Seslerio autumnalis-Fagetum and (or) Lamio orvalae-Fagetum.

Viola pyrenaica on the southern edge of the Trnovski gozd plateau was found to grow in communities which are physiognomically similar to those elsewhere in the Julian Alps (tall herb communities, hop hornbeam coppice stands - compare Table 4 with Tables 1 and 3); the latter, however, are significantly different regarding their floristic composition. Communities in the Julian Alps have more diagnostic species from classes Elyno-Sesleriet $e a$ and Mulgedio-Aconitetea, and beech forest species from the order Fagetalia sylvaticae grow more abundantly within them. On the southern edge of the Trnovski gozd plateau, these communities consist of considerably more Illyrian-sub-Mediterranean species, character and differential species of the order Scorzoneretalia villosae (= Scorzonero-Chrysopogonetalia); also more abundant are some of the diagnostic species of the classes Trifolio-Geranietea and (because of even rockier sites) Asplenietea trichomanis, and of the order Quercetalia pubescentis.

The new locality of Viola pyrenaica on the slopes of the Nanos plateau above Podnanos is entirely in sub-Mediterranean phytogeographical region of Slovenia. Here it thrives in sub-Mediterranean fringe community, together with species Anthericum ramosum, Asparagus tenuifolius, Carex humilis, Centaurea triumfettii, Coronilla emerus subsp. emeroides, Cotinus coggygria, Dianthus monspessulanus, Dictamnus albus, Genista sericea, G. sylvestris, Inula hirta, I. spiraeifolia, Iris pallida subsp. illyrica, Polygonatum odoratum, Seseli gouanii, Thalictrum minus etc.

\section{DISGUSSION AND CONGLUSIONS}

Numerous new localities of the south-European montane species Viola pyrenaica were found in the course of floristic and vegetation research of the last several years in the Julian Alps. In our opinion, this species, at least in the Soča region of this mountain range, is not as rare and endangered as we used to believe (Wraber \& Skoberne 1989: 336, Wraber et al. 2002: 8910). It is more likely that its localities get overlooked because of the plant's early blooming, as well as on account of the fact that it is often overshadowed by other plants, most often tall herbs, during the vegetation season.
Similar factors may be the reason why this species has been overlooked also in the northern part of the Dinaric mountains, where for the time being its occurrence is determined only on the southern edge of the Trnovski gozd plateau between Col and Otlica above the Vipava Valley and on the slopes of the Nanos plateau (southwestern Slovenia). In this region, it grows on similar sites and in (physiognomically) similar plant communities to those in the Julian Alps (in the submontane and montane belt, on calcareous bedrock, on stony grasslands which are being overgrown with tall herbs, in stony hop hornbeam - Ostrya carpinifolia and flowering ash - Fraxinus ornus forests, on forest edges), but with a considerably different floristic composition. These communities are therefore classified into other associations and higher syntaxonomical units (grasslands that are being overgrown are treated as successional stages on the sites of the association Genisto sericeae-Seslerietum kalnikensis from the alliance Satureion subspicatae; stony forests, on the other hand, are classified into the association Seslerio autumnalis-Ostryetum and into the alliance Carpinion orientalis).

In the Julian Alps and on the southern edge of the Trnovski gozd and Nanos plateaus, Viola pyrenaica has so far been found within the communities of the following higher synsystematic units: class Elyno-Seslerietea (alliance Caricion austroalpinae); class Festuco-Brometea (alliance Mesobromion = Bromion erecti, alliance Satureion subspicatae), class Erico-Pinetea (alliance Erico-Fraxinion orni) and class Querco-Fagetea (order Quercetalia pubescentis, alliance Carpinion orientalis; order Fagetalia sylvaticae, alliance Aremonio-Fagion, suballiance OstryoFagenion); its occurrence has also been determined in the succession stages similar to the communities from the classes Mulgedio-Aconitetea (above all from the order Adenostyletalia), Thlaspietea rotundifolii (order Stipetalia calamagrostis, alliance Stipion calamagrostis) and Trifolio-Geranietea (within the latter to the communities of the alliance Geranion sanguinei and, in the Trnovski gozd and Nanos plateaus, suballiance Dictamno-Ferulagenion). Because it occurs within different communities it is difficult to characterize it as a diagnostic species of one the above-mentioned higher synsystematic units. We have established that the sites of Viola pyrenaica on the localities known so far in Slovenia are similar to those in the other parts of the Alps and the Dinaric mountains; these sites are above all overgrown screes, stony grasslands, forest edges, light stony forests, tall herb communities in the 
submontane, montane, altimontane and subalpine belt (450 to $1600 \mathrm{~m}$ a.s.l.).

\section{POVZETEK}

Vrsta Viola pyrenaica Ramond ex DC v severnem delu Dinarskega gorstva (Trnovski gozd in Nanos, Slovenija)

Pri florističnih in vegetacijskih raziskavah v letih 2006 in 2007 smo v Julijskih Alpah našli precej novih nahajališč južnoevropske gorske vrste Viola pyrenaica: v južnih Julijskih Alpah, v soseščini Črne prsti pod Lajnarjem, Slatnikom, Šavnikom in Liscem ter v Trtniku nad Podbrdom; v Bohinju na levem bregu Save Bohinjke pod Rsnikom in pod Stadorjem nad Črnim jezerom; v Krnskem pogorju v Morizni pod grebenom Polovnika in na Bovškem v dolinah Možnice, Učje in Lepene. Menimo, da to vsaj v posoškem delu tega gorovja ni zelo redka in ogrožena vrsta, kar smo mislili doslej (Wraber \& Skoberne 1989: 336, Wraber et al. 2002: 8910), pač pa njena nahajališča zaradi zgodnjega pomladanskega cvetenja in ker jo v glavni vegetacijski sezoni prerasejo druge rastline, pogosto visoke steblike, najbrž večkrat spregledamo.

Morda smo iz podobnih razlogov ta vrsto doslej spregledali tudi v severnem delu Dinarskega gorstva, kjer za zdaj ugotavljamo le njeno pojavljanje na južnem robu Trnovskega gozda med Colom in Otlico nad Vipavsko dolino - 0149/2 (UTM VL18) ter na pobočjih Nanosa nad Podnanosom (0150/3, UTM VL27). Na južnem robu Trnovskega gozda, na in pod grebenom Sončnica-Podrta gora-Leskov školj, uspeva na podobnih rastiščih in $\mathrm{v}$ fiziognomsko (po videzu) podobnih rastlinskih združbah kot v Julijskih Alpah: v montanskem pasu, na karbonatni podlagi, na kamnitih traviščih, ki se zaraščajo z visokimi steblikami, v kamnitih gozdovih črnega gabra in malega jesena in na gozdnih robovih. Toda floristična sestava teh združb je precej drugačna, zato jih uvrščamo $\mathrm{v}$ druge asociacije in višje sintaksonomske enote. Zaraščajoča travišča obravnavamo kot sukcesijske stadije na rastiščih asociacije Genisto sericeae-Seslerietum kalnikensis iz zveze Satureion subspicatae, kamnite gozdove pa uvrščamo v asociacijo Seslerio autumnalis-Ostryetum in v zvezo Carpinion orientalis. Pod Nanosom smo pirenejsko vijolico popisali na kamnitem robu submediteranskega panjevskega gozda črnega gabra in puhastega hrasta (Ostryo-Quercetum pubescentis), v združbi z vrstami Anthericum ram- osum, Asparagus tenuifolius, Carex humilis, Centaurea triumfettii, Coronilla emerus subsp. emeroides, Cotinus coggygria,Dianthus monspessulanus, Dictamnus albus, Genista sericea, G. sylvestris, Inula hirta, I. spiraeifolia, Iris pallida subsp. illyrica, Polygonatum odoratum, Seseli gouanii,Thalictrum minus idr.

V Julijskih Alpah in v severnem delu Dinarskega gorstva smo doslej vrsto Viola pyrenaica našli v združbah naslednjih višjih sinsistematskih enot: razred Elyno-Seslerietea (zveza Caricion austroalpinae); razred Festuco-Brometea (zveza Mesobromion oz. Bromion erecti, zveza Satureion subspicatae), razred Erico-Pinetea (zveza Erico-Fraxinion orni) in razred Querco-Fagetea (red Quercetalia pubescentis, zveza Carpinion orientalis; red Fagetalia sylvaticae, zveza Aremonio-Fagion, podzveza Ostryo-Fagenion), prav tako smo ugotovili njeno pojavljanje $\mathrm{v}$ sukcesijskih stadijih, ki so podobni združbam iz razredov Mulgedio-Aconitetea (predvsem reda Adenostyletalia), Thlaspietea rotundifolii (iz reda Stipatalia calamagrostis in zveze Stipion calamagrostis) in TrifolioGeranietea (v slednjem združbam zveze Geranion sanguinei in, v Trnovskem gozdu in pod Nanosom, podvzveze Dictamno-Ferulagenion). Zaradi različnih združb, v katerih se pojavlja, jo je težko označiti kot diagnostično vrsto katere od naštetih višjih sinsistematskih enot.Ugotavljamo, da so rastišča vrste Viola pyrenaica na doslej znanih nahajališčih v Sloveniji podobna tistim v drugih delih Alp in Dinarskega gorstva, torej predvsem porasel grušč, kamnita travišča, gozdni robovi, svetli kamniti gozdovi, združbe visokih steblik v submontanskem, montanskem, altimontanskem in subalpinskem pasu (450 do $1600 \mathrm{~m}$ n. m. v.).

\section{ACKNOWLEDGEMENTS}

For her valuable help in preparation of this article, the first author would like to thank Dr. Željka Bjelčić and Dr. Boštjan Surina who kindly provided data on the sites of Viola pyrenaica in Bosnia and Herzegovina. Mag. Andrej Seliškar determined some grass species (especially from the genus Festuca). Academician Prof. Dr. Ernest Mayer, Prof. Dr. Tone Wraber, Prof. Dr. Vladimir Stevanović, Prof. Dr. Vlado Matevski, Prof. Dr. Mitko Kostadinovski, Dr. Branko Vreš, Dr. Toni Nikolić, Olga DežmanJokić, Ivan Veber and Branko Zupan helped us with their advice, literature and other information - sincere thanks to all of them. English translation was provided by Andreja Šalamon Verbič. 


\section{REFERENCES}

Aeschimann, D., Lauber, K., Moser, D. M. \& Theurillat, J.-P. 2004: Flora alpina. Bd. 1: Lycopodiaceae-Apiaceae. Haupt Verlag, Bern, Stuttgart, Wien, $1159 \mathrm{pp}$.

Aichele, D. \& Schwegler, H.-W. 1995: Die Blütenpflanzen Mitteleuropas. Band 3. Franckh-Kosmos, Stuttgart, $576 \mathrm{pp}$.

Beck, G. 1916: Flora Bosne, Hercegovine i bivšeg Sandžaka Novog Pazara. II. dio (8. nastavak). Glasnik Zemaljskog muzeja u Bosni i Hercegovini (Sarajevo) 28: 353-393.

Beck, G. 1919: Flora Bosne, Hercegovine i bivšeg Sandžaka Novog Pazara. Glasnik Zemaljskog muzeja u Bosni i Hercegovini (Sarajevo) 30 (1918): 177-218.

Braun-Blanquet,J. 1964: Pflanzensoziologie. Grundzüge der Vegetationskunde. 3. Auf., Wien-New York, Springer, 865 pp.

Bjelčić, Ž. 2006: Viola pyrenaica u Bosni i Hercegovini. Manuscript, Sarajevo, 2 pp.

Dakskobler, I. 2005: Floristične novosti iz Posočja in sosednjih območij v zahodni in severozahodni Sloveniji - IV. Hacquetia 4 (2): 173-200.

Dakskobler, I., Franz, W. R., Seljak, G. 2005: Communities with Eryngium alpinum in the southern Julian Alps (Mts. Crna prst and Porezen). Hacquetia (Ljubljana) 4 (2): 83-120.

Diklić, N. 1972: Violaceae. In: M. Josifović (ed.): Flora SR Srbije III, Srpska akademija nauka i umetnosti, Beograd, pp. 128-164.

Ehrendorfer, F. \& Hamman, U. 1965: Vorschläge zu einer floristischen Kartierung von Mitteleuropa. Ber. Deutsch. Bot. Ges. 78: 35-50.

Erben, M. 1995: Viola L. In: Micevski, K.: Flora na Republika Makedonija (The Flora of the Republic of Macedonia). Vol. 1, Book 3. Makedonska akademija na naukite i umetnostite, Skopje, pp. 503-548.

Frahm, J. P. \& Frey, W. 1992: Moosflora. 3. Aufl. UTB, Eugen Ulmer, Stuttgart, 528 pp.

Gams, H. \& Becker, W. 1925: Violaceae. In: Hegi, G.: Ilustrierte Flora von Mittel-Europa. 1. Aufl. Band V (1), Carl Hanser, München, pp. 585-668.

Hayek, A. 1927: Prodromus Florae peninsulae Balcanicae. Band I. Reprint with a new introduction by W. T. Steran, 1975. Otto Koeltz Science Publishers, Koenigstein, 1193 pp.

Hess, E. H., Landolt, E. \& Hirzel, R. 1977: Flora der Schweiz und angrenzender Gebiete. Band 2, 2. Aufl., Birkhäuser, Basel, Stuttgart, 956 pp.

Kos, V. (ed.) 1996: Atlas Slovenije. Tretja izpopol- njena in razširjena izdaja. Mladinska knjiga, Ljubljana, 440 pp.

Maarel van der, E. 1979: Transformation of coverabundance values in phytosociology and its effects on community similarity. Vegetatio 39 (2): 97-114.

Martinčič, A. 2003: Seznam listnatih mahov (Bryopsida) Slovenije. Hacquetia 2 (1): 91-166.

Martinčič, A., Wraber, T., Jogan, N., Ravnik, V., Podobnik, A., Turk, B. \& Vreš, B. 1999: Mala flora Slovenije. Ključ za določanje praprotnic in semenk. Tehniška založba Slovenije, Ljubljana, $845 \mathrm{pp}$.

Mayer, E. 1954: Kritični prispevki k flori slovenskega ozemlja II. Razprave 4. razreda SAZU (Ljubljana) 2: 5-44.

Meusel, H., Jäger, E., Rauschert, S. \& Weinert, E. 1978: Vergleichende Chorologie der Zentraleuropäischen Flora. Band II. Text. Gustav Fischer Verlag, Jena 418 pp.

Pignatti, S. 1982: Flora d'Italia. Vol. 2. Edagricole, Bologna, 732 pp.

Podani, J. 2001: SYN-TAX 2000. Computer Programs for Data Analysis in Ecology and Systematics. User's Manual, Budapest, 53 pp.

Qosja, X. (ed.) 1992: Flora e Shqiperise, Bd. 2 (Flore de l'Albanie, 2). Akademia e Shkencave e Republikes se Shqiperise, Qendra e kerkimeve biologjike, Tirana, $466 \mathrm{pp}$.

Seliškar, T., Vreš, B. \& Seliškar, A. 2003: FloVegSi 2.0. Računalniški program za urejanje in analizo bioloških podatkov. Biološki inštitut ZRC SAZU, Ljubljana.

Strid, A. (ed.) 1989: Mountain Flora of Greece. Vol. 1. Cambridge University Press, Cambridge, $822 \mathrm{pp}$.

Surina, B. 2004: Carex austroalpina Becherer, a new southeastern-Alpine species for the flora of Slovenia, and Viola pyrenaica Ramond ex DC., second record for the flora of the Julian Alps. Annales Ser. hist. nat. 14 (2): 231-236.

Šegulja, N. 1997: Violaceae. In: Nikolić, T. (ed.): Flora Croatica. Index florae Croaticae. Pars 2. Natura Croatica 6, Suppl. 1, pp. 17-18.

Trinajstić, I. 1975: Porod. Violaceae. In: Trinajstić, I. (ed.): Analitička flora Jugoslavije 2 (1). Inštitut za botaniku Sveučilišta u Zagrebu, Zagreb, pp. 34-79.

Valentine, D. H., Merxmüller, H. \& Schmidt, A. 1968: Viola L. In: Tutin, T. G. et al. (eds.): Flora Europaea. Volume 2: Rosaceae to Umbelliferae. University Press, Cambridge, pp. 270-282.

Wraber, M. 1969: Pflanzengeographische Stel- 
lung und Gliederung Sloweniens. Vegetatio 17: 176-199.

Wraber, T. \& Skoberne, P. 1989: Rdeči seznam ogroženih praprotnic in semenk SR Slovenije. Varstvo narave 14-15: 1-429.

Wraber, T., Skoberne, P., Seliškar, A., Vreš, B., Babij, V., Čušin, B., Dakskobler, I., Surina, B., Šilc, U., Zelnik, I., Žagar, V., Jogan, N., Leskovar, I., Kaligarič, M. \& Bavcon J. 2002: Pravilnik o uvrstitvi ogroženih rastlinskih in živalskih vrst v rdeči seznam. Priloga 1: Rdeči seznam praprotnic in semenk (Pteridophyta \& Spermatophyta). Uradni list RS 12 (82): 8893-8910, Ljubljana.

\section{APPENDIX}

List of syntaxa mentioned in the article with authors:

Asplenietea trichomanis Br.-Bl. in Meier \& Br.-Bl. 1934

Thlaspietea rotundifolii $\mathrm{Br}$.-Bl. in Br.-Bl. \& Jenny 1926

Stipetalia calamagrostis Oberdorfer \& Seibert in Oberdorfer 1977 ( = Galio-Parietarietalia officinalis Boşcaiu et al. 1966)

Stipion calamagrostis Jenny-Lips ex Quantin 1932

Koelerio-Corynephoretea Klika in Klika \& Novák 1941 (= Sedo-Scleranthetea Br.-Bl. 1955)

Molinio-Arrhenatheretea R. Tx. 1937 em. R. Tx. 1970

Poo alpinae-Trisetalia Ellmauer \& Mucina 1993

Calluno-Ulicetea Br.-Bl. \& R. Tx. ex Klika 1948

Festuco-Brometea Br.-Bl. \& Tx. 1943

Mesobromion erecti (Br.-Bl. \& Moor 1938)

Oberdorfer 1957 = Bromion erecti Br.-Bl.

(1925) 1936

Scorzoneretalia villosae Horvatić 1975 ( = Scorzonero-Chrysopogonetalia Horvatić \& Horvat in Horvatić 1958)

Satureion subspicatae Horvat 1962

Genisto sericeae-Seslerietum juncifoliae Poldini 1980 var. geogr. Sesleria kalnikensis Poldini 2005 = Genisto sericeae-Seslerietum kalnikensis

Elyno-Seslerietea Br.- Bl. 1948

Caricion austroalpinae Sutter 1962

Centaureo julicae-Laserpitetum sileris Dakskobler 2003 nom. prov.
Eryngio alpinae-Caricetum ferrugineae Seljak ex Dakskobler, Franz \& Seljak 2005

Avenastro parlatorei-Festucetum calvae Aichinger 1933 corr. Franz 1980

Seslerietalia juncifoliae Horvat 1930

Festucetum bosniacae Horvat 1930

Trifolio-Geranietea Th. Müller 1962

Origanetalia vulgaris Th. Müller 1962

Geranion sanguinei Tüxen in Th. Müller 1962

Dictamno-Ferulagenion van Gils, Keysers \&

Launspach 1975

Epilobietea angustifolii R. Tx. \& Preising in R. Tx. 1950

Mulgedio-Aconitetea Hadač \& Klika in Klika 1948

Adenostyletalia G. \&. J. Br.-Bl. 1931

Erico-Pinetea I. Horvat 1959

Erico-Fraxinion orni Horvat 1959

Ostryo carpinifoliae-Fraxinetum orni Aichinger

1933 - Fraxino orni-Ostryetum carpinifoliae

Aichinger 1933 corr. Franz 2002

Cytisantho-Ostryetum M. Wraber 1961

Vaccinio-Piceetea Br.-Bl. 1939 emend. Zupančič (1976) 2000

Chrysanthemo rotundifoliae-Piceenion (Krajina

1933) Theurillat in Aeschimann et al. 2004

Rhamno-Prunetea Rivas Goday \& Borja Carbonell 1961

Querco-Fagetea Br.-Bl. \& Vlieg. 1937

Fagetalia sylvaticae Pawł. in Pawł. et al. 1928

Aremonio-Fagion (Ht. 1938) Borhidi in Török,

Podani \& Borhidi 1989

Lamio orvalae-Fagetum (Ht. 1938) Borhidi 1963

Ranunculo platanifolii-Fagetum Marinček et al. 1993

Ostryo-Fagenion Borhidi 1963

Ostryo-Fagetum M. Wraber ex Trinajstić 1972

Seslerio autumnalis-Fagetum (Ht.) M. Wraber ex Borhidi 1963

Quercetalia pubescentis Klika 1933

Ostryo-Carpinion orientalis Horvat 1954 em. 1958

Seslerio albicantis-Ostryetum Lausi et al. 1982 corr. Poldini et Vidali 1995

Seslerio autumnalis-Ostryetum I. Horvat \& Horvatić 1950 corr. Zupančič 1999

Ostryo-Quercetum pubescentis (Ht. 1950)

Trinajstić 1974

Quercetalia roboris-petraeae R. Tx. 1931 


\section{ABBREVIATIONS - OKRAJŠAVE}

\section{Parent material (Geološka podlaga)}

A - limestone - apnenec

$\mathrm{Gr}$ - talus - pobočni grušč

Mo - moraine (til) - morena (til)

\section{Soil types (Talni tipi)}

$\mathrm{R}$ - rendzina - rendzina

Li - Lithosols - litosol

Tables (Tabele) 1-4

Table 1: Tal herbs communities with Viola pyrenaica in the southern Julian Alps

Tabela 1: Združbe visokih steblik z vrsto Viola pyrenaica v južnih Julijskih Apah

Number of relevé (Številka popisa)

Working number (Delovna št. popisa)

Altitude in $\mathrm{m}$ (Nadmorska višina v m)

Aspect (Lega)

Slope in degrees (Nagib v stopinjah)

Parent material (Matična podlaga)

Soil (Tla)

Stoniness in \% (Kamnitost v \%)

Cover in \% (Zastiranje v \%):

Shrub layer (Grmovna plast)

Herb layer (Zeliščna plast)

Relevé area (Velikost popisne ploskve)

Number of species (Število vrst)

Date of taking relevé (Datum popisa)

Locality (Nahajališče)

Quadrant (Kvadrant)

\begin{tabular}{cccccc}
1 & 2 & 3 & 4 & 5 & 6 \\
\hline 212047 & 212394 & 212389 & 212395 & 212390 & 212396 \\
1600 & 1440 & 1470 & 1500 & 1470 & 1520 \\
SE & SW & SSW & SW & SW & SW \\
10 & 40 & 40 & 40 & 45 & 35 \\
A & A & A & A & A & A \\
R & R & R & R & R & R \\
0 & 10 & 10 & 5 & 5 & 10
\end{tabular}

$\begin{array}{ccccccc}\mathrm{E} 2 & 10 & \cdot & \cdot & \cdot & \cdot & \cdot \\ \mathrm{E} 1 & 90 & 100 & 100 & 100 & 100 & 100 \\ \mathrm{~m}^{2} & 15 & 20 & 20 & 20 & 20 & 20 \\ & 41 & 34 & 52 & 59 & 45 & 55\end{array}$

8/9/2006 7/14/2005 7/14/2005 7/14/2005 7/14/2005 7/14/2005

Lisec Šavnik Šavnik Šavnik Šavnik Šavnik

9749/4 9749/4 9749/4 9749/4 9749/4 9749/4

Pr. Fr.

\section{Caricion austroalpinae}

Centaurea haynaldii subsp. julica

Festuca calva

$\begin{array}{llll}\text { E1 } & + & + & 1 \\ \text { E1 } & \cdot & + & 1 \\ \text { E1 } & \cdot & \cdot & + \\ \text { E1 } & + & \cdot & \cdot \\ & & & \\ \text { E1 } & 1 & 1 & 1 \\ \text { E1 } & + & 1 & 1 \\ \text { E1 } & + & 1 & 1 \\ \text { E1 } & \text { r } & \cdot & 1 \\ \text { E1 } & \cdot & + & + \\ \text { E1 } & \cdot & \cdot & 1 \\ \text { E1 } & \cdot & \cdot & + \\ \text { E1 } & \cdot & \cdot & \cdot\end{array}$




\begin{tabular}{|c|c|c|c|c|c|c|c|c|c|}
\hline Number of relevé (Številka popisa) & & 1 & 2 & 3 & 4 & 5 & 6 & Pr. & Fr. \\
\hline Potentilla crantzii & E1 & . & . & . & + & . & + & 2 & 33 \\
\hline Cerastium strictum & E1 & . & . & . & . & + & + & 2 & 33 \\
\hline Rhinanthus glacialis & E1 & + & . & . & . & . & . & 1 & 17 \\
\hline Acinos alpinus & E1 & . & . & . & + & . & . & 1 & 17 \\
\hline Ranunculus montanus & E1 & . & . & . & . & + & . & 1 & 17 \\
\hline Anthyllis vulneraria subsp. alpestris & E1 & . & . & . & . & . & + & 1 & 17 \\
\hline Carex sempervirens & E1 & . & . & . & . & . & 1 & 1 & 17 \\
\hline Galium anisophyllon & E1 & & . & . & . & . & + & 1 & 17 \\
\hline Polygala alpestris & E1 & . & . & . & . & . & + & 1 & 17 \\
\hline Thesium alpinum & E1 & . & . & . & . & . & + & 1 & 17 \\
\hline \multicolumn{10}{|l|}{ Mulgedio-Aconitetea } \\
\hline Silene vulgaris subsp. antelopum & E1 & 1 & 1 & + & 1 & + & + & 6 & 100 \\
\hline Hypericum maculatum & E1 & . & + & + & + & + & + & 5 & 83 \\
\hline Serratula tinctoria subsp. macrocephala & E1 & 1 & . & 2 & 1 & + & 2 & 5 & 83 \\
\hline Viola pyrenaica & E1 & 1 & . & + & + & + & + & 5 & 83 \\
\hline Polygonatum verticillatum & E1 & 3 & . & + & . & . & + & 3 & 50 \\
\hline Lathyrus occidentalis var. montanus & E1 & + & . & . & + & . & + & 3 & 50 \\
\hline $\begin{array}{l}\text { Aconitum lycoctonum subsp. } \\
\text { ranunculifolium }\end{array}$ & E1 & . & . & + & 1 & . & + & 3 & 50 \\
\hline Astrantia major & E1 & . & . & + & + & . & + & 3 & 50 \\
\hline Pimpinella major subsp. rubra & E1 & . & . & + & + & . & + & 3 & 50 \\
\hline Veratrum album & E1 & . & . & + & + & . & + & 3 & 50 \\
\hline Eryngium alpinum & E1 & 2 & . & . & + & . & . & 2 & 33 \\
\hline Rumex alpestris & E1 & + & . & . & + & . & . & 2 & 33 \\
\hline Calamagrostis arundinacea & E1 & . & + & + & . & . & . & 2 & 33 \\
\hline Poa hybrida & E1 & 1 & . & . & . & . & . & 1 & 17 \\
\hline Sorbus chamaemespilus & E1 & 1 & . & . & . & . & . & 1 & 17 \\
\hline Carduus carduelis & E1 & + & . & . & . & . & . & 1 & 17 \\
\hline Crepis pyrenaica & E1 & + & . & . & . & . & . & 1 & 17 \\
\hline Heracleum montanum & E1 & + & . & . & . & . & . & 1 & 17 \\
\hline Myrrhis odorata & E1 & + & . & . & . & . & . & 1 & 17 \\
\hline Thalictrum aquilegiifolium & E1 & + & . & . & . & . & . & 1 & 17 \\
\hline Allium victorialis & E1 & . & . & . & + & . & . & 1 & 17 \\
\hline \multicolumn{10}{|l|}{ Trifolio-Geranietea s. lat. } \\
\hline Laserpitium siler & E1 & 3 & 3 & 4 & 3 & 4 & 4 & 6 & 100 \\
\hline Iris graminea & E1 & + & + & + & 1 & + & + & 6 & 100 \\
\hline Arabis pauciflora & E1 & + & . & + & + & + & + & 5 & 83 \\
\hline Thalictrum minus & E1 & . & 1 & 1 & + & + & + & 5 & 83 \\
\hline Silene nutans & E1 & . & . & + & + & + & + & 4 & 67 \\
\hline Polygonatum odoratum & E1 & . & 1 & + & . & 1 & . & 3 & 50 \\
\hline Peucedanum schottii & E1 & . & + & + & . & + & . & 3 & 50 \\
\hline Laserpitium latifolium & E1 & . & . & + & 1 & . & . & 2 & 33 \\
\hline Tanacetum corymbosum subsp. clusii & E1 & . & . & + & + & . & . & 2 & 33 \\
\hline Lilium carniolicum & E1 & . & . & + & . & + & . & 2 & 33 \\
\hline Origanum vulgare & E1 & + & . & . & . & . & . & 1 & 17 \\
\hline Vicia sylvatica & E1 & + & . & . & . & . & . & 1 & 17 \\
\hline Vincetoxicum hirundinaria & E1 & . & 1 & . & . & . & . & 1 & 17 \\
\hline Valeriana collina & E1 & . & . & . & + & . & . & 1 & 17 \\
\hline
\end{tabular}


Number of relevé (Številka popisa)

2

3

4

5

6

Pr. Fr.

Buphthalmum salicifolium

E1

Carex humilis

E1

$+$

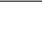

Bromus erectus agg.

Gymnadenia conopsea

Koeleria pyramidata agg.

Prunella grandiflora

Thlaspi praecox

Linum viscosum

Allium senescens

Hippocrepis comosa

Carlina acaulis subsp. caulescens

Centaurea triumfettii

\section{Poo alpinae-Trisetalia}

Phleum hirsutum

Festuca nigrescens

Traunsteinera globosa

Trollius europaeus

Ranunculus nemorosus

Molinio-Arrhenatheretea

Galium mollugo

Lotus corniculatus

Dactylis glomerata

Vicia sepium

Trifolium pratense

E1

E1

E1

E1

E1

E1

E1

E1

E1

E1

$+$

$\begin{array}{rrrr}+ & + & 6 & 100 \\ 2 & 1 & 5 & 83 \\ 1 & 1 & 5 & 83 \\ + & + & 5 & 83 \\ 2 & 1 & 5 & 83 \\ + & + & 3 & 50 \\ + & . & 3 & 50 \\ . & . & 2 & 33 \\ 1 & . & 2 & 33 \\ + & . & 2 & 33 \\ . & + & 2 & 33 \\ + & . & 1 & 17\end{array}$

Calluno-Ulicetea

Phyteuma zahlbruckneri

Anthoxanthum odoratum

Carex pallescens

E1

E1

E1

E1

E1

1

$$
83
$$

$$
83
$$$$
83
$$$$
50
$$

$$
33
$$$$
+\quad+\quad 583
$$$$
\begin{array}{llll}
1 & 1 & 5 & 83
\end{array}
$$$$
\begin{aligned}
& 1 \\
& +\quad 1 \quad 53
\end{aligned}
$$$$
\text { . } \quad+\quad 233
$$$$
\begin{array}{r}
2 \\
+\quad 17
\end{array}
$$$$
\mathrm{E} 1+\quad+\quad+\quad+\quad+\quad+\quad+\quad 6 \quad+\quad 600
$$

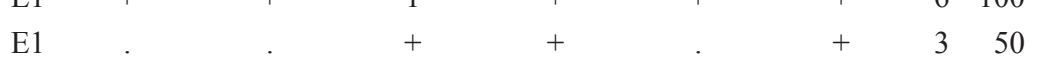

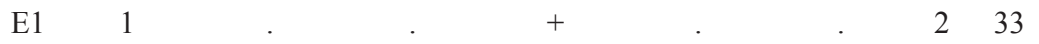

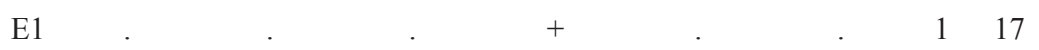$$
\text { E1 }
$$$$
17
$$

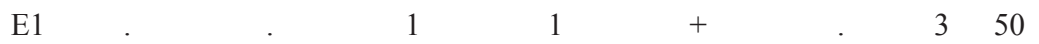$$
\text { E1 . }
$$

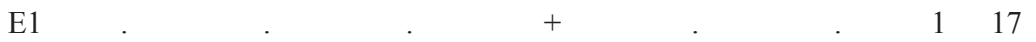

\section{Erico-Pinetea}

Cirsium erisithales

Peucedanum austriacum var. rablense

Genista radiata

Calamagrostis varia

Chamaecytisus hirsutus subsp. ciliatus

$\begin{array}{lllllllll}\text { E1 } & 1 & . & 1 & 1 & + & 1 & 5 & 83 \\ \text { E1 } & + & . & + & 1 & + & + & 5 & 83 \\ \text { E1 } & 3 & 4 & . & . & . & . & 2 & 33 \\ \text { E1 } & . & 2 & . & . & + & . & 2 & 33 \\ \text { E1 } & . & . & + & . & . & . & 1 & 17\end{array}$

\section{Fagetalia sylvaticae}

Mercurialis perennis

Knautia drymeia

Symphytum tuberosum

E1 1

E1

E1

$\mathrm{E} 1+$

Acer pseudoplatanus

E1 +

Galium laevigatum

E1

E1

Poa nemoralis

E1

Lilium martagon

E1

.

Querco-Fagetea s. lat.

Ornithogalum pyrenaicum

E1

$+$

1

1

$\begin{array}{rrrr}1 & 1 & 6 & 100 \\ + & + & 5 & 83 \\ + & \cdot & 2 & 33 \\ . & \cdot & 1 & 17 \\ . & \cdot & 1 & 17 \\ . & . & 1 & 17 \\ . & . & 1 & 17 \\ . & . & 1 & 17 \\ . & \cdot & 1 & 17 \\ & & & \\ + & + & 6 & 100\end{array}$




\begin{tabular}{|c|c|c|c|c|c|c|c|c|c|}
\hline Number of relevé (Številka popisa) & & 1 & 2 & 3 & 4 & 5 & 6 & Pr. & Fr. \\
\hline Carex digitata & E1 & + & . & + & . & . & . & 2 & 33 \\
\hline Luzula luzuloides & E1 & . & . & . & + & . & . & 1 & 17 \\
\hline Anemone nemorosa & E1 & . & . & . & . & . & + & 1 & 17 \\
\hline \multicolumn{10}{|l|}{ Asplenietea trichomanis } \\
\hline Sedum maximum & E1 & + & + & . & . & . & . & 2 & 33 \\
\hline Primula auricula & E1 & . & . & . & . & + & + & 2 & 33 \\
\hline Saxifraga hostii & E1 & . & . & . & . & . & + & 1 & 17 \\
\hline \multicolumn{10}{|l|}{ Other species (Druge vrste) } \\
\hline Rubus idaeus & E1 & . & + & . & + & . & . & 2 & 33 \\
\hline
\end{tabular}

Table 2: Communities with Viola pyrenaica on overgrown screes in the Možnica valley (the Julian Alps, the Bovec region)

Tabela 2: Združbe z vrsto Viola pyrenaica na poraslih meliščih v dolini Možnice (Julijske Alpe, Bovško)

\begin{tabular}{|c|c|c|c|c|c|c|}
\hline & Number of relevé (Številka popisa) & & 1 & 2 & 3 & \\
\hline & Working number (Delovna št. popisa) & & 212377 & 212378 & 212379 & \\
\hline & Altitude in $\mathrm{m}$ (Nadmorska višina $\mathrm{v} \mathrm{m}$ ) & & 930 & 940 & 1050 & \\
\hline & Aspect (Lega) & & SE & SE & SE & \\
\hline & Slope in degrees (Nagib v stopinjah) & & 25 & 30 & 30 & \\
\hline & Parent material (Matična podlaga) & & Gr & Gr & Gr & \\
\hline & Soil (Tla) & & $\mathrm{Li}$ & $\mathrm{Li}$ & $\mathrm{Li}$ & \\
\hline & Stoniness in \% (Kamnitost v \%) & & 60 & 30 & 30 & \\
\hline & \multicolumn{6}{|l|}{ Cover in \% (Zastiranje v \%): } \\
\hline & Herb layer (Zeliščna plast) & E1 & 60 & 70 & 70 & \\
\hline & Relevé area (Velikost popisne ploskve) & $\mathrm{m}^{2}$ & 10 & 20 & 20 & \\
\hline & Number of species (Število vrst) & & 25 & 48 & 49 & \\
\hline & Date of taking relevé (Datum popisa) & & $6 / 26 / 2006$ & $6 / 26 / 2006$ & $6 / 26 / 2006$ & \\
\hline & Locality (Nahajališče) & & Možnica & Možnica & Možnica & \\
\hline & Quadrant (Kvadrant) & & $9647 / 1$ & $9647 / 1$ & $9647 / 1$ & \\
\hline & \multicolumn{6}{|c|}{ Character species of the alliance Stipion calamagrostis } \\
\hline & Geranium macrorrhizum & E1 & . & + & 2 & 2 \\
\hline & Scrophularia juratensis & E1 & . & + & . & 1 \\
\hline & \multicolumn{6}{|c|}{ Differential species of the alliance Stipion calamagrostis } \\
\hline FB & Galium lucidum & E1 & $\mathrm{r}$ & 1 & 1 & 3 \\
\hline TG & Vincetoxicum hirundinaria & E1 & 1 & . & 2 & 2 \\
\hline TG & Origanum vulgare & E1 & + & . & . & 1 \\
\hline AT & Sedum album & E1 & . & + & . & 1 \\
\hline \multicolumn{7}{|c|}{ Thlaspietea rotundifolii s. lat. } \\
\hline & Iris pallida subsp. cengialti & E1 & 1 & 2 & 3 & 3 \\
\hline & Viola pyrenaica & E1 & 2 & + & 1 & 3 \\
\hline & Aconitum lycoctonum subsp. ranunculifolium & E1 & . & 1 & 1 & 2 \\
\hline & Heracleum pollinianum & E1 & . & + & + & 2 \\
\hline & Moehringia muscosa & E1 & + & . & . & 1 \\
\hline & Rumex scutatus & E1 & . & + & . & 1 \\
\hline & Silene vulgaris subsp. glareosa & E1 & . & + & . & 1 \\
\hline
\end{tabular}


Number of relevé (Številka popisa)

Gymnocarpium robertianum

Polystichum lonchitis

Festuco-Brometea

Euphorbia cyparissias

Bromus erectus agg.

Ajuga genevensis

Teucrium chamaedrys

Arabis hirsuta

Anthyllis vulneraria

Peucedanum oreoselinum

Carlina acaulis

Galium purpureum

Buphthalmum salicifolium

Carex humilis

Stachys recta

Trifolio-Geranietea

Clinopodium vulgare

Thalictrum minus

Verbascum alpinum

Silene nutans

Valeriana collina

Laserpitium latifolium

Verbascum lychnitis

Digitalis grandiflora

Laserpitium siler

Thesium bavarum

Elyno-Seslerietea

Carduus crassifolius

Betonica alopecuros

Acinos alpinus

Campanula witasekiana

Cerastium strictum

Festuca calva

Thymus praecox subsp. polytrichus

Molinio-Arrhenatheretea

Dactylis glomerata

Veronica chamaedrys

Lotus corniculatus

Ranunculus sardous

Mulgedio-Aconitetea

Hesperis candida

Silene vulgaris subsp. antelopum

Silene dioica

Veratrum album

Epilobietea angustifoliae

Galeopsis speciosa
E1

E1

E1

E1
E1
E1

1

$\begin{array}{cc}3 & \text { Pr. } \\ + & 1 \\ + & 1 \\ + & 1\end{array}$

E1

E1

E1

E1

E1

E1

E1

E1

E1

E1

E1

E1

E1

E1

E1

E1

E1

E1

E1

E1

E1

E1

E1

E1

E1

E1

E1

E1

E1

E1

E1

E1

E1

E1

E1

E1

E1

E1

$+$ 


\begin{tabular}{|c|c|c|c|c|c|}
\hline Number of relevé (Številka popisa) & & 1 & 2 & 3 & Pr. \\
\hline Carex muricata & E1 & . & + & . & 1 \\
\hline Fragaria vesca & E1 & . & . & + & 1 \\
\hline \multicolumn{6}{|l|}{ Galio-Urticetea } \\
\hline Urtica dioica & E1 & + & + & . & 2 \\
\hline Fallopia dumetorum & E1 & + & . & . & 1 \\
\hline Lamium maculatum & E1 & . & + & . & 1 \\
\hline \multicolumn{6}{|l|}{ Quercetalia pubescentis } \\
\hline Primula veris subsp. columnae & E1 & . & 1 & 1 & 2 \\
\hline Rosa glauca & E1 & . & + & + & 2 \\
\hline Arabis turrita & E1 & + & . & . & 1 \\
\hline Fraxinus ornus & E1 & + & . & . & 1 \\
\hline Peucedanum schottii & E1 & . & 1 & . & 1 \\
\hline Clematis recta & E1 & . & + & . & 1 \\
\hline Sorbus aria & E2 & . & . & + & 1 \\
\hline \multicolumn{6}{|l|}{ Fagetalia sylvaticae } \\
\hline Campanula trachelium & E1 & + & . & + & 2 \\
\hline Salvia glutinosa & E1 & . & + & + & 2 \\
\hline Geranium robertianum & E1 & + & . & . & 1 \\
\hline Brachypodium sylvaticum & E1 & + & . & . & 1 \\
\hline Myosotis sylvatica & E1 & . & + & . & 1 \\
\hline Acer pseudoplatanus & E1 & . & . & + & 1 \\
\hline Galium laevigatum & E1 & . & . & 1 & 1 \\
\hline Melica nutans & E1 & . & . & 1 & 1 \\
\hline Mercurialis perennis & E1 & . & . & 1 & 1 \\
\hline Cardamine enneaphyllos & E1 & . & . & + & 1 \\
\hline Cyclamen purpurascens & E1 & . & . & + & 1 \\
\hline Fraxinus excelsior & E1 & . & . & + & 1 \\
\hline Galeobdolon flavidum & E1 & . & . & + & 1 \\
\hline \multicolumn{6}{|l|}{ Querco-Fagetea } \\
\hline Clematis vitalba & E1 & . & + & . & 1 \\
\hline Carex digitata & E1 & . & . & + & 1 \\
\hline \multicolumn{6}{|l|}{ Erico-Pinetea } \\
\hline Aster amellus & E1 & . & + & . & 1 \\
\hline Carex ornithopoda & E1 & . & . & + & 1 \\
\hline Cirsium erisithales & E1 & . & . & + & 1 \\
\hline
\end{tabular}


Table 3: Ostrya carpinifolia stands with Viola pyrenaica in the Julian Alps (the Bovec region)

Tabela 3: Sestoji črnega gabra z vrsto Viola pyrenaica v Julijskih Alpah (Bovško)

\begin{tabular}{|c|c|c|c|c|c|c|c|c|c|c|c|c|}
\hline Number of relevé (Številka popisa) & & 1 & 2 & 3 & 4 & 5 & 6 & 7 & 8 & 9 & & \\
\hline Working number (Delovna št. popisa) & & $\begin{array}{l}\tilde{\infty} \\
\stackrel{\tilde{N}}{\sim} \\
\stackrel{\sim}{N}\end{array}$ & 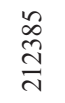 & $\begin{array}{l}\hat{\infty} \\
\underset{\sim}{\sim} \\
\stackrel{\sim}{N}\end{array}$ & $\begin{array}{l}\stackrel{+}{\infty} \\
\underset{\sim}{\sim} \\
\underset{\sim}{N}\end{array}$ & $\begin{array}{l}\infty \\
\infty \\
\stackrel{\sim}{\sim} \\
\stackrel{\sim}{N}\end{array}$ & 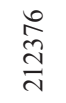 & $\begin{array}{l}\vec{\infty} \\
\underset{\sim}{\sim} \\
\vec{\sim}\end{array}$ & $\begin{array}{l}\stackrel{\infty}{\infty} \\
\underset{\sim}{\sim}\end{array}$ & $\begin{array}{l}\infty \\
\infty \\
\underset{\sim}{\sim} \\
\underset{\sim}{\Delta}\end{array}$ & & \\
\hline Altitude in m (Nadmorska višina $\mathrm{v}$ m) & & 490 & 620 & 610 & 590 & 660 & 440 & 440 & 470 & 810 & & \\
\hline Aspect (Lega) & & SSE & SW & SW & SW & SE & $\mathrm{S}$ & $\mathrm{S}$ & $\mathrm{S}$ & SW & & \\
\hline Slope in degrees (Nagib v stopinjah) & & 45 & 45 & 45 & 30 & 35 & 30 & 30 & 45 & 10 & & \\
\hline Parent material (Matična podlaga) & & A & A & A & Gr & A & Gr & Gr & A & Mo & & \\
\hline Soil (Tla) & & $\mathrm{R}$ & $\mathrm{R}$ & $\mathrm{R}$ & $\mathrm{R}$ & $\mathrm{R}$ & $\mathrm{R}$ & $\mathrm{R}$ & $\mathrm{R}$ & $\mathrm{R}$ & & \\
\hline Stoniness in \% (Kamnitost $\mathrm{v} \%)$ & & 60 & 30 & 50 & 20 & 30 & 30 & 60 & 70 & 30 & & \\
\hline \multicolumn{13}{|l|}{ Cover in \% (Zastiranje v \%): } \\
\hline Upper tree layer (Zgornja drevesna plast) & $\mathrm{E} 3 \mathrm{~b}$ & 70 & 80 & 60 & 90 & 70 & 90 & 80 & 70 & 90 & & \\
\hline Lower tree layer (Spodnja drevesna plast) & E3a & . & . & . & . & 20 & . & 10 & 10 & . & & \\
\hline Shrub layer (Grmovna plast) & E2 & 40 & 30 & 50 & 40 & 30 & 30 & 30 & 40 & 10 & & \\
\hline Herb layer (Zeliščna plast) & E1 & 60 & 70 & 70 & 70 & 70 & 70 & 60 & 60 & 70 & & \\
\hline Moss layer (Mahovna plast) & E0 & 10 & 10 & 5 & 10 & 10 & 20 & 40 & 30 & 20 & & \\
\hline Maximum diameter (Največji prsni premer) & $\mathrm{cm}$ & 20 & 25 & 20 & 30 & 25 & 25 & 25 & 25 & 20 & & \\
\hline Maximum height (Največja drevesna višina) & $\mathrm{m}$ & 8 & 10 & 8 & 17 & 16 & 12 & 15 & 10 & 14 & & \\
\hline Relevé area (Velikost popisne ploskve) & $\mathrm{m}^{2}$ & 200 & 400 & 200 & 400 & 200 & 200 & 400 & 200 & 400 & & \\
\hline Number of species (Število vrst) & & 77 & 89 & 54 & 87 & 68 & 86 & 75 & 69 & 53 & & \\
\hline Date of taking relevé (Datum popisa) & & 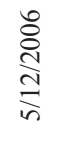 & 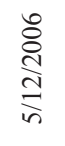 & 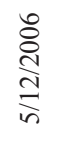 & 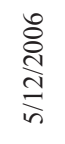 & 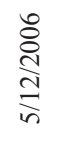 & 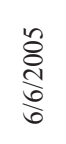 & 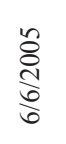 & 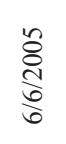 & 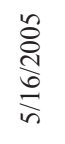 & & \\
\hline Locality (Nahajališče) & & $\begin{array}{l}\stackrel{\pi}{\tilde{N}} \\
\stackrel{0}{0} \\
\Sigma\end{array}$ & 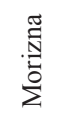 & $\begin{array}{l}\text { Nี } \\
\stackrel{\tilde{0}}{\Sigma}\end{array}$ & 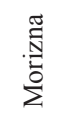 & 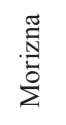 & $\begin{array}{l}\frac{\pi}{8} \\
\frac{\pi}{0} \\
0 \\
2\end{array}$ & $\begin{array}{l}\frac{\pi}{0} \\
\frac{\pi}{0} \\
0 \\
0\end{array}$ & $\begin{array}{l}\frac{\pi}{\mathbb{N}} \\
\frac{70}{0} \\
0\end{array}$ & $\frac{\pi}{\pi}$ & & \\
\hline Quadrant (Kvadrant) & & 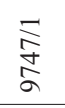 & 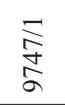 & 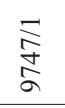 & 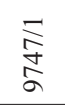 & 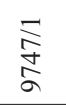 & 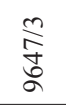 & 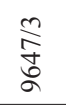 & $\frac{m}{\stackrel{d}{d}}$ & 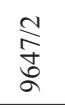 & & \\
\hline \multicolumn{11}{|l|}{ Ostryo-Carpinion orientalis } & Pr. & \\
\hline Ostrya carpinifolia & $\mathrm{E} 3 \mathrm{~b}$ & 3 & 4 & 3 & 4 & 3 & 5 & 5 & 4 & 4 & 9 & $\mathrm{~V}$ \\
\hline Ostrya carpinifolia & E3a & . & . & . & . & . & . & + & . & . & 1 & I \\
\hline Ostrya carpinifolia & $\mathrm{E} 2 \mathrm{~b}$ & . & . & + & . & . & + & . & . & . & 2 & II \\
\hline Ostrya carpinifolia & E2a & + & . & . & . & . & . & . & . & . & 1 & I \\
\hline Ostrya carpinifolia & E1 & . & . & . & . & . & . & + & . & . & 1 & I \\
\hline Coronilla emerus subsp. emeroides & $\mathrm{E} 2 \mathrm{a}$ & 1 & 1 & 1 & . & . & . & . & . & . & 3 & II \\
\hline Frangula rupestris & E2a & . & . & 1 & . & . & . & . & . & . & 1 & I \\
\hline Frangula rupestris & E1 & . & . & + & . & . & . & . & . & . & 1 & I \\
\hline \multicolumn{13}{|l|}{ Quercetalia pubescentis } \\
\hline Fraxinus ornus & $\mathrm{E} 3 \mathrm{~b}$ & 3 & 2 & 2 & 2 & 2 & 1 & 1 & 3 & + & 9 & $\mathrm{~V}$ \\
\hline Fraxinus ornus & E3a & . & 1 & . & 1 & 1 & . & . & . & . & 3 & II \\
\hline Fraxinus ornus & $\mathrm{E} 2 \mathrm{~b}$ & 1 & 1 & 1 & 1 & . & 2 & 2 & 2 & 1 & 8 & $\mathrm{~V}$ \\
\hline Fraxinus ornus & E2a & 2 & 2 & 1 & 3 & 1 & 2 & 2 & 2 & . & 8 & $\mathrm{~V}$ \\
\hline Fraxinus ornus & E1 & . & 1 & . & . & + & 1 & . & + & . & 4 & III \\
\hline Cornus mas & $\mathrm{E} 2 \mathrm{~b}$ & 2 & 1 & + & 2 & 1 & + & . & + & . & 7 & IV \\
\hline Cornus mas & $\mathrm{E} 2 \mathrm{a}$ & + & . & . & . & + & . & + & + & . & 4 & III \\
\hline Cornus mas & E1 & . & . & . & . & . & + & . & . & . & 1 & I \\
\hline Sorbus aria & E3b & . & 1 & . & . & 1 & . & . & . & . & 2 & II \\
\hline Sorbus aria & E3a & . & + & . & + & . & . & . & . & . & 2 & II \\
\hline
\end{tabular}




\begin{tabular}{|c|c|c|c|c|c|c|c|c|c|c|c|c|}
\hline Number of relevé (Številka popisa) & & 1 & 2 & 3 & 4 & 5 & 6 & 7 & 8 & 9 & Pr. & $\mathrm{Cl}$. \\
\hline Sorbus aria & $\mathrm{E} 2 \mathrm{~b}$ & . & . & + & + & + & . & + & + & . & 5 & III \\
\hline Sorbus aria & $\mathrm{E} 2 \mathrm{a}$ & + & . & . & + & . & + & + & . & . & 4 & III \\
\hline Sorbus aria & E1 & . & + & . & + & . & . & . & . & + & 3 & II \\
\hline Arabis turrita & E1 & 1 & 1 & . & 1 & 1 & + & . & . & . & 5 & III \\
\hline Melittis melissophyllum & E1 & 1 & 1 & 1 & 1 & 1 & . & . & . & . & 5 & III \\
\hline Euonymus verrucosa & E2a & . & + & + & + & 1 & . & . & . & . & 4 & III \\
\hline Peucedanum schottii & E1 & + & . & . & + & . & . & . & + & + & 4 & III \\
\hline Tamus communis & E1 & + & . & + & + & + & . & . & . & . & 4 & III \\
\hline Campanula persicifolia & E1 & + & + & . & 1 & . & . & . & . & . & 3 & II \\
\hline Clematis recta & E1 & + & . & + & . & . & . & . & . & . & 2 & II \\
\hline Convallaria majalis & E1 & . & + & . & . & + & . & . & . & . & 2 & II \\
\hline Primula veris subsp. columnae & E1 & . & . & . & . & . & + & . & . & 1 & 2 & II \\
\hline Tanacetum corymbosum & E1 & + & . & . & . & . & . & . & . & . & 1 & I \\
\hline Hypericum montanum & E1 & . & + & . & . & . & . & . & . & . & 1 & I \\
\hline Quercus pubescens & E1 & . & + & . & . & . & . & . & . & . & 1 & I \\
\hline \multicolumn{13}{|l|}{ Aremonio-Fagion } \\
\hline Cyclamen purpurascens & E1 & 1 & 1 & 1 & 1 & + & 1 & + & + & 1 & 9 & $\mathrm{~V}$ \\
\hline Anemone trifolia & E1 & + & . & . & 1 & 1 & . & . & . & . & 3 & II \\
\hline Helleborus niger & E1 & + & + & . & . & 1 & . & . & . & . & 3 & II \\
\hline Cardamine enneaphyllos & E1 & . & + & . & . & . & . & . & . & . & 1 & $\mathrm{I}$ \\
\hline \multicolumn{13}{|l|}{ Fagetalia sylvaticae } \\
\hline Campanula trachelium & E1 & + & + & . & 1 & + & 1 & 1 & + & . & 7 & IV \\
\hline Salvia glutinosa & E1 & $\mathrm{r}$ & + & . & + & . & + & + & . & + & 6 & IV \\
\hline Brachypodium sylvaticum & E1 & . & . & . & + & + & + & + & . & 1 & 5 & III \\
\hline Galium laevigatum & E1 & + & + & . & 1 & 1 & . & . & . & . & 4 & III \\
\hline Lathyrus vernus & E1 & + & 1 & . & + & + & . & . & . & . & 4 & III \\
\hline Asarum europaeum subsp. caucasicum & E1 & + & . & . & 1 & 1 & . & . & . & . & 3 & II \\
\hline Acer pseudoplatanus & E1 & . & $\mathrm{r}$ & . & . & + & . & . & . & + & 3 & II \\
\hline Mercurialis perennis & E1 & . & . & . & + & 1 & . & . & . & + & 3 & II \\
\hline Mycelis muralis & E1 & . & . & . & + & . & + & + & . & . & 3 & II \\
\hline Neottia nidus-avis & E1 & . & . & . & + & + & . & + & . & . & 3 & II \\
\hline Dryopteris filix-mas & E1 & . & . & . & + & . & + & + & . & . & 3 & II \\
\hline Carpinus betulus & $\mathrm{E} 3 \mathrm{~b}$ & . & . & . & + & . & . & . & . & . & 1 & I \\
\hline Carpinus betulus & E3a & . & $\mathrm{r}$ & . & . & . & . & . & . & . & 1 & I \\
\hline Carpinus betulus & $\mathrm{E} 2 \mathrm{a}$ & . & . & . & . & . & . & + & . & . & 1 & I \\
\hline Carpinus betulus & E1 & . & $\mathrm{r}$ & . & + & . & . & . & . & . & 2 & II \\
\hline Tilia cordata & E3a & . & $\mathrm{r}$ & . & . & . & . & . & . & . & 1 & I \\
\hline Tilia cordata & $\mathrm{E} 2 \mathrm{a}$ & . & + & . & . & . & . & + & . & . & 2 & II \\
\hline Tilia cordata & E1 & . & . & . & . & . & + & . & . & . & 1 & I \\
\hline Fraxinus excelsior & $\mathrm{E} 3 \mathrm{~b}$ & . & . & . & $\mathrm{r}$ & . & . & . & . & 1 & 2 & II \\
\hline Fraxinus excelsior & E2a & . & . & . & . & . & . & . & . & 1 & 1 & I \\
\hline Fraxinus excelsior & E1 & . & . & . & . & . & . & . & . & 1 & 1 & I \\
\hline Galeobdolon flavidum & E1 & . & . & . & + & . & . & . & . & + & 2 & II \\
\hline Tilia platyphyllos & $\mathrm{E} 3 \mathrm{~b}$ & . & . & . & . & + & . & . & . & . & 1 & I \\
\hline Tilia platyphyllos & E3a & . & $\mathrm{r}$ & . & . & . & . & . & . & . & 1 & $\mathrm{I}$ \\
\hline Tilia platyphyllos & $\mathrm{E} 2 \mathrm{~b}$ & . & + & . & . & . & . & . & . & + & 2 & II \\
\hline Tilia platyphyllos & $\mathrm{E} 2 \mathrm{a}$ & . & . & . & . & . & . & . & . & + & 1 & I \\
\hline Tilia platyphyllos & E1 & . & . & . & . & + & + & . & . & . & 2 & II \\
\hline Fagus sylvatica & $\mathrm{E} 3 \mathrm{~b}$ & . & . & . & . & . & . & . & . & + & 1 & I \\
\hline Fagus sylvatica & E3a & . & $\mathrm{r}$ & . & . & . & . & . & . & . & 1 & I \\
\hline Fagus sylvatica & $\mathrm{E} 2 \mathrm{a}$ & . & . & . & . & . & . & . & . & + & 1 & I \\
\hline
\end{tabular}


Number of relevé (Številka popisa)

Fagus sylvatica

Euphorbia amygdaloides

Laburnum alpinum

Myosotis sylvatica

Polygonatum multiflorum

Prunus avium

Senecio fuchsii

Acer platanoides

Acer platanoides

Daphne mezereum

Quercetalia roboris

Hieracium racemosum

Festuca heterophylla

Quercus robur

Frangula alnus

Frangula alnus

Pteridium aquilinum

Melampyrum pratense

Chamaecytisus supinus

Querco-Fagetea

Viola riviniana

Carex digitata

Vinca minor

Corylus avellana

Corylus avellana

Corylus avellana

Hepatica nobilis

Clematis vitalba

Clematis vitalba

Primula vulgaris

Lonicera xylosteum

Acer campestre

Acer campestre

Acer campestre

Acer campestre

Quercus petraea

Cephalanthera longifolia

Cruciata glabra

Helleborus odorus

Veratrum nigrum

Geum urbanum

Hedera helix

Pyrus pyraster

Viola mirabilis

Dactylis polygama

Listera ovata

Rhamno-Prunetea

Rhamnus catharticus

Crataegus monogyna

Crataegus monogyna

E1

E1

E2b

E1

E1

E2a

E1

E2a

E1

E2a

E1

E1

E1

E2a

E1

E1

E1

E1

E1

$2 a$

b

$2 a$

$\begin{array}{lllllllllll}1 & 2 & 3 & 4 & 5 & 6 & 7 & 8 & 9 & \text { Pr. } \quad \mathrm{Cl}\end{array}$

. . . . . . . . . .

(1)

(1)

a

a

$2 \mathrm{a}$

$+$

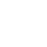

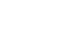

. . . . . . . . . .

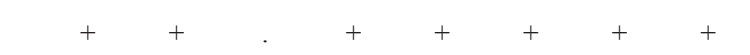

$$
\text { V }
$$$$
\text { IV }
$$$$
\text { IV }
$$$$
\text { I }
$$$$
\text { III }
$$$$
\text { I }
$$$$
\text { III }
$$$$
\text { III }
$$$$
\text { I }
$$$$
\text { III }
$$$$
3 \text { II }
$$$$
\text { I }
$$$$
2 \text { II }
$$$$
1 \text { I }
$$$$
2 \text { II }
$$$$
2 \text { II }
$$$$
2 \text { II }
$$$$
\begin{aligned}
& +.2 . \\
& +
\end{aligned}
$$

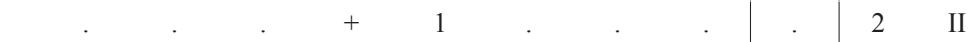

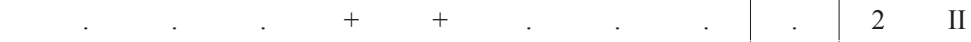$$
\begin{array}{lllllll}
\cdot & + & + & \cdot & \cdot & 2 & \text { II }
\end{array}
$$$$
\text { . . . . . . . . . } 1 \text { I }
$$ 


\begin{tabular}{|c|c|c|c|c|c|c|c|c|c|c|c|c|}
\hline Number of relevé (Številka popisa) & & 1 & 2 & 3 & 4 & 5 & 6 & 7 & 8 & 9 & Pr. & $\mathrm{Cl}$. \\
\hline Ligustrum vulgare & E2a & + & . & . & . & . & + & + & + & . & 4 & III \\
\hline Ligustrum vulgare & E1 & . & . & . & . & . & + & . & . & . & 1 & I \\
\hline Cornus sanguinea & $\mathrm{E} 2 \mathrm{~b}$ & . & . & . & . & . & . & + & . & . & 1 & I \\
\hline Cornus sanguinea & $\mathrm{E} 2 \mathrm{a}$ & . & . & . & + & . & . & . & . & . & 1 & I \\
\hline Cornus sanguinea & E1 & . & . & . & . & . & + & . & . & . & 1 & I \\
\hline Euonymus europaea & E2a & . & . & . & . & + & . & . & . & . & 1 & I \\
\hline Rhamnus saxatilis & E2a & . & . & . & . & . & . & . & + & . & 1 & I \\
\hline Rosa glauca & E2a & . & . & + & . & . & . & . & . & . & 1 & I \\
\hline Rosa canina & E2a & . & . & . & . & . & . & . & + & . & 1 & I \\
\hline Viburnum lantana & E2a & . & . & . & . & . & + & . & . & . & 1 & I \\
\hline Berberis vulgaris & $\mathrm{E} 2 \mathrm{a}$ & . & . & . & . & . & . & . & + & . & 1 & I \\
\hline \multicolumn{13}{|l|}{ Trifolio-Geranietea } \\
\hline Silene nutans & E1 & 1 & + & + & + & + & + & + & 1 & . & 8 & $\mathrm{~V}$ \\
\hline Campanula rapunculoides & E1 & + & + & + & 1 & 1 & + & + & . & . & 7 & IV \\
\hline Clinopodium vulgare & E1 & + & + & . & 1 & + & + & 1 & 1 & . & 7 & IV \\
\hline Libanotis sibirica subsp. montana & E1 & + & + & + & + & + & + & . & 1 & . & 7 & IV \\
\hline Vincetoxicum hirundinaria & E1 & 1 & + & 1 & . & + & . & + & + & + & 7 & IV \\
\hline Viola hirta & E1 & + & + & 1 & + & + & + & . & 1 & . & 7 & IV \\
\hline Anthericum ramosum & E1 & + & 1 & 1 & + & + & . & . & + & . & 6 & IV \\
\hline Polygonatum odoratum & E1 & . & + & 1 & + & + & . & + & . & + & 6 & IV \\
\hline Digitalis grandiflora & E1 & + & + & . & + & + & . & . & . & . & 4 & III \\
\hline Valeriana collina & E1 & + & + & . & + & + & . & . & . & . & 4 & III \\
\hline Lilium carniolicum & E1 & $\mathrm{r}$ & + & . & + & . & . & . & . & . & 3 & II \\
\hline Thesium bavarum & E1 & + & + & + & . & . & . & . & . & . & 3 & II \\
\hline Thalictrum minus & E1 & . & . & + & + & . & . & . & . & . & 2 & II \\
\hline Trifolium rubens & E1 & . & . & . & + & . & . & . & + & . & 2 & II \\
\hline Laserpitium latifolium & E1 & . & . & . & . & + & . & . & . & + & 2 & II \\
\hline Lembotropis nigricans & E1 & . & . & . & . & . & + & . & + & . & 2 & II \\
\hline Verbascum lychnitis & E1 & . & . & . & . & . & + & . & + & . & 2 & II \\
\hline Origanum vulgare & E1 & 1 & . & . & . & . & . & . & . & . & 1 & I \\
\hline Inula conyza & E1 & . & . & . & + & . & . & . & . & . & 1 & I \\
\hline Valeriana nemorensis & E1 & . & . & . & + & . & . & . & . & . & 1 & I \\
\hline Achillea distans & E1 & . & . & . & . & . & . & + & . & . & 1 & I \\
\hline Astragalus glycyphyllos & E1 & . & . & . & . & . & . & + & . & . & 1 & I \\
\hline \multicolumn{13}{|l|}{ Festuco-Brometea } \\
\hline Carex humilis & E1 & 3 & 1 & 1 & 1 & + & 3 & 2 & 1 & . & 8 & $\mathrm{~V}$ \\
\hline Galium purpureum & E1 & + & + & 1 & + & . & + & + & 1 & . & 7 & IV \\
\hline Buphthalmum salicifolium & E1 & . & + & + & . & . & 1 & + & 1 & + & 6 & IV \\
\hline Dianthus monspessulanus & E1 & 1 & 1 & . & + & . & 1 & 1 & 1 & . & 6 & IV \\
\hline Genista tinctoria & E1 & + & 1 & . & + & . & + & 1 & 1 & . & 6 & IV \\
\hline Peucedanum oreoselinum & E1 & + & + & + & . & . & + & + & + & . & 6 & IV \\
\hline Teucrium chamaedrys & E1 & 1 & + & 1 & + & . & + & . & + & . & 6 & IV \\
\hline Allium carinatum & E1 & + & . & . & + & + & . & . & + & + & 5 & III \\
\hline Arabis hirsuta & E1 & + & + & . & . & . & 1 & + & . & + & 5 & III \\
\hline Euphorbia cyparissias & E1 & . & + & 1 & + & . & + & + & . & . & 5 & III \\
\hline Galium lucidum & E1 & . & . & + & + & . & + & + & + & . & 5 & III \\
\hline Ajuga genevensis & E1 & 1 & + & . & + & . & . & . & + & . & 4 & III \\
\hline Brachypodium rupestre & E1 & + & . & . & . & . & 1 & . & + & . & 3 & II \\
\hline Bromus erectus agg. & E1 & 1 & . & . & + & . & . & . & + & . & 3 & II \\
\hline Satureja montana subsp. variegata & E1 & + & . & 1 & . & . & . & . & + & . & 3 & II \\
\hline
\end{tabular}


Number of relevé (Številka popisa)

Scabiosa triandra

Centaurea triumfettii

Stachys recta

Medicago lupulina

Pimpinella saxifraga

Helianthemum ovatum

Allium carinatum subsp. pulchellum

Poa angustifolia

Carlina vulgaris

Hippocrepis comosa

Dactylorhiza sambucina

Molinio-Arrhenatheretea

Veronica chamaedrys

Achillea millefolium

Galium mollugo

Centaurea jacea

Dactylis glomerata

Lathyrus pratensis

Phyteuma zahlbruckneri

Lotus corniculatus

Trifolium pratense

Ranunculus nemorosus

Taraxacum officinale

Elyno-Seslerietea

Festuca calva

Sesleria caerulea subsp. calcaria

Acinos alpinus

Carduus crassifolius

Betonica alopecuros

Phyteuma orbiculare

Asperula aristata

Ranunculus carinthiacus

\section{Erico-Pinetea}

Aster amellus

Calamagrostis varia

Polygala chamaebuxus

Epipactis atrorubens

Carex ornithopoda

Chamaecytisus hirsutus

Genista radiata

Carex alba

Amelanchier ovalis

Amelanchier ovalis

Leontodon incanus

Peucedanum austriacum var. rablense

Vaccinio-Piceetea

Solidago virgaurea

Hieracium murorum

Picea abies

Oxalis acetosella

E1

E1

E1

E1

E1

E1

E1

E1

E1

E1

E1

E1

E1

E1

E1

E1

E1

E1

E1

E1

E1

E1

E1

E1

E1

E1

E1

E1

E1

E1

E1

E1

E1

E1

E1

E1

E2a

E1

E2b

E2a

E1

E1

E1

E1

E1

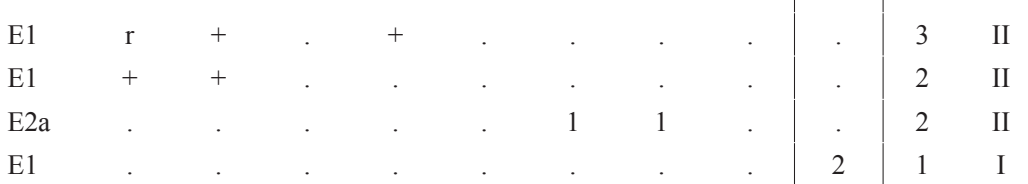

\begin{tabular}{lllllllll|lll}
1 & 2 & 3 & 4 & 5 & 5 & 6 & 7 & 8 & 9 & Pr. $\mathrm{Cl}$
\end{tabular}

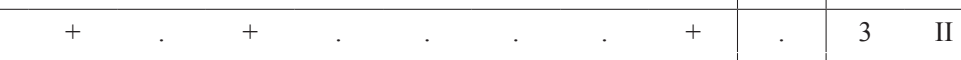

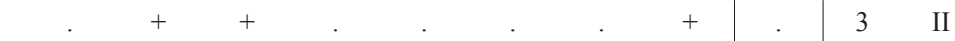

$\begin{array}{lllllllll}1 & + & . & . & . & . & . & 2 & \end{array}$

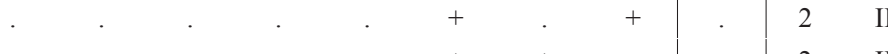

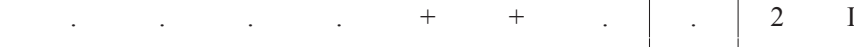

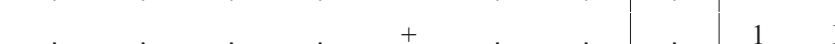

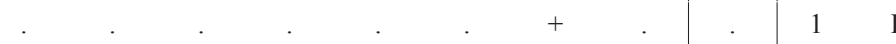

\begin{tabular}{lll|l|l|l}
+ & + & + & + & 4
\end{tabular}

\begin{tabular}{llllll|ll}
. & + & + & + & + & & &
\end{tabular}

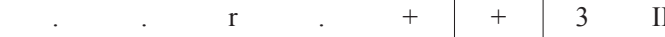

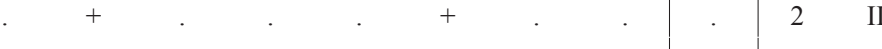

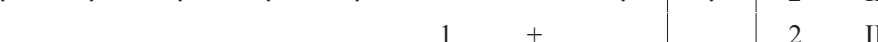

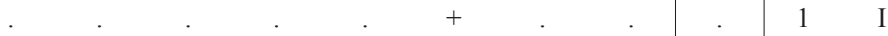

\begin{tabular}{llll|l|ll}
$\mathrm{r}$ & $\mathrm{r}$ & + & + & + & &
\end{tabular}

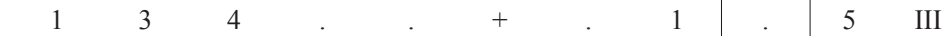

\begin{tabular}{rrrr|r|rr}
+ & + & + & &
\end{tabular}

$\begin{array}{llllllllll}+ & + & & + & + & +\end{array}$

IV

IV

II

3 II

II

II

II

II

I

I

1 I 


\begin{tabular}{|c|c|c|c|c|c|c|c|c|c|c|c|c|}
\hline Number of relevé (Številka popisa) & & 1 & 2 & 3 & 4 & 5 & 6 & 7 & 8 & 9 & Pr. & $\mathrm{Cl}$. \\
\hline Picea abies & E1 & . & . & . & . & . & . & . & . & + & 1 & $\mathrm{I}$ \\
\hline \multicolumn{13}{|l|}{ Thlaspietea rotundifolii s. lat. } \\
\hline Viola pyrenaica & E1 & + & . & + & . & + & + & 1 & . & 1 & 6 & IV \\
\hline Hieracium glaucum & E1 & . & + & . & . & . & + & + & + & . & 4 & III \\
\hline Hieracium porrifolium & E1 & + & + & + & . & . & . & . & . & . & 3 & II \\
\hline Hieracium bifidum & E1 & . & . & + & . & . & 1 & 1 & . & . & 3 & II \\
\hline Aconitum angustifolium & E1 & . & . & . & + & . & . & . & . & . & 1 & I \\
\hline Hesperis candida & E1 & . & . & . & . & + & . & . & . & . & 1 & I \\
\hline Geranium macrorrhizum & E1 & . & . & . & . & . & . & 1 & . & . & 1 & I \\
\hline Aconitum lycoctonum & E1 & . & . & . & . & . & . & . & . & 1 & 1 & I \\
\hline \multicolumn{13}{|l|}{ Asplenietea trichomanis s. lat. } \\
\hline Asplenium ruta-muraria & E1 & 1 & 1 & + & + & 1 & 1 & 1 & 1 & + & 9 & $\mathrm{~V}$ \\
\hline Moehringia muscosa & E1 & 1 & + & 1 & 1 & 1 & 1 & 1 & + & . & 8 & $\mathrm{~V}$ \\
\hline Asplenium trichomanes & E1 & 1 & + & . & + & 1 & 1 & 1 & 1 & . & 7 & IV \\
\hline Campanula carnica & E1 & + & + & + & . & . & + & + & 1 & . & 6 & IV \\
\hline Polypodium vulgare & E1 & . & + & . & + & + & 1 & 1 & . & . & 5 & III \\
\hline Sedum album & E1 & . & + & . & . & . & + & + & + & . & 4 & III \\
\hline Sedum maximum & E1 & + & . & . & + & . & . & + & . & . & 3 & II \\
\hline Campanula spicata & E1 & . & + & + & . & . & . & . & + & . & 3 & II \\
\hline Ceterach officinarum s. lat. & E1 & . & . & + & . & . & . & . & + & . & 2 & II \\
\hline Festuca stenantha & E1 & . & . & . & . & . & + & . & 1 & . & 2 & II \\
\hline Sedum hispanicum & E1 & . & . & . & . & . & . & 1 & + & . & 2 & II \\
\hline Asplenium adiantum-nigrum & E1 & . & . & . & . & . & + & . & . & . & 1 & I \\
\hline Saxifraga hostii & E1 & . & . & . & . & . & . & . & + & . & 1 & I \\
\hline Silene hayekiana & E1 & . & . & . & . & . & . & . & + & . & 1 & $\mathrm{I}$ \\
\hline \multicolumn{13}{|l|}{ Other species (Druge vrste) } \\
\hline Fragaria vesca & E1 & 1 & + & . & 1 & 1 & 1 & + & + & + & 8 & $\mathrm{~V}$ \\
\hline Juniperus communis & $\mathrm{E} 2 \mathrm{~b}$ & . & . & . & . & . & . & + & 1 & . & 2 & II \\
\hline Juniperus communis & E2a & . & . & . & . & . & + & 1 & 1 & . & 3 & II \\
\hline Juniperus communis & E1 & . & . & . & . & . & + & + & . & . & 2 & II \\
\hline Torilis japonica & E1 & . & . & . & . & r & . & + & . & . & 2 & II \\
\hline Festuca sp. & E1 & + & . & . & . & . & . & . & . & . & 1 & I \\
\hline Rubus caesius & E2a & . & . & . & + & . & . & . & . & . & 1 & $\mathrm{I}$ \\
\hline Juglans regia & E1 & . & . & . & . & . & + & . & . & . & 1 & I \\
\hline \multicolumn{13}{|l|}{ Mosses and lichens (Mahovi in lišaji) } \\
\hline Homalothecium sericeum & E0 & 1 & + & + & 1 & . & + & . & + & + & 7 & IV \\
\hline Anomodon viticulosus & E0 & + & + & . & 1 & + & . & 2 & 2 & . & 6 & IV \\
\hline Schistidium apocarpum & E0 & 1 & + & + & 1 & . & . & . & 1 & + & 6 & IV \\
\hline Homalothecium lutescens & E0 & . & . & . & 1 & . & 2 & 2 & 2 & 1 & 5 & III \\
\hline Ctenidium molluscum & E0 & . & + & . & + & + & . & 1 & . & . & 4 & III \\
\hline Hypnum cupressiforme & E0 & . & . & . & 1 & . & + & 1 & . & 1 & 4 & III \\
\hline Peltigera canina & E0 & . & . & . & + & . & . & + & + & . & 3 & II \\
\hline Porella platyphylla & E0 & . & . & . & . & . & + & + & + & . & 3 & II \\
\hline Tortella tortuosa & E0 & 1 & . & . & . & . & . & . & . & + & 2 & II \\
\hline Neckera crispa & E0 & . & 1 & . & . & + & . & . & . & . & 2 & II \\
\hline Anomodon attenuatus & E0 & . & . & . & + & 1 & . & . & . & . & 2 & II \\
\hline Isothecium alopecuroides & E0 & . & . & . & + & 1 & . & . & . & . & 2 & II \\
\hline Rhytidium rugosum & E0 & . & + & . & . & . & . & . & . & . & 1 & I \\
\hline Cladonia pyxidata & E0 & . & . & . & + & . & . & . & . & . & 1 & I \\
\hline Thuidium delicatulum & E0 & . & . & . & . & . & . & + & . & . & 1 & I \\
\hline
\end{tabular}


Table 4: Communities with Viola pyrenaica on the southern edge of the Trnovski gozd plateau

Tabela 4: Združbe z vrsto Viola pyrenaica na južnem robu Trnovskega gozda

\begin{tabular}{|c|c|c|c|c|c|c|c|c|c|c|c|c|c|c|}
\hline \multirow[b]{2}{*}{ Number of relevé (Številka popisa) } & & \multicolumn{9}{|c|}{ I } & \multicolumn{4}{|c|}{ II } \\
\hline & & 1 & 2 & 3 & 4 & 5 & 6 & 7 & 8 & 9 & 10 & 11 & 12 & 13 \\
\hline Working number (Delovna št. popisa) & & $\begin{array}{l}\stackrel{ \pm}{\approx} \\
\stackrel{\sim}{\sim}\end{array}$ & $\underset{\tilde{n}}{\stackrel{n}{N}}$ & 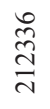 & $\begin{array}{l}\hat{\tilde{\Xi}} \\
\stackrel{\sim}{\sim}\end{array}$ & $\begin{array}{l}\stackrel{+}{+} \\
\underset{\sim}{\sim}\end{array}$ & 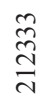 & $\begin{array}{l}\stackrel{?}{\tilde{N}} \\
\stackrel{\sim}{\sim}\end{array}$ & 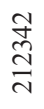 & $\frac{\mathfrak{n}}{\stackrel{\sim}{\sim}}$ & $\begin{array}{l}\vec{J} \\
\stackrel{\sim}{v}\end{array}$ & 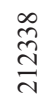 & $\begin{array}{l}\text { ले } \\
\underset{\sim}{\sim}\end{array}$ & $\begin{array}{l}\stackrel{\forall}{\sim} \\
\stackrel{\sim}{\sim}\end{array}$ \\
\hline Altitude in $\mathrm{m}$ (Nadmorska višina $\mathrm{v} \mathrm{m}$ ) & & ஓ & $\stackrel{\infty}{\infty}$ & $\underset{\infty}{\infty}$ & $\underset{\infty}{\infty}$ & $\underset{\infty}{\infty}$ & ஓ & $\underset{\infty}{\infty}$ & ஓ & $\stackrel{\infty}{\infty}$ & $\underset{\infty}{\infty}$ & $\frac{P}{\infty}$ & $\underset{\infty}{\stackrel{2}{\infty}}$ & $\stackrel{?}{\infty}$ \\
\hline Aspect (Lega) & & 嵴 & $\sqrt[W]{n}$ & is & $\sum_{i=2}^{3}$ & $\sum_{i s}^{3}$ & 㞱 & 㭊 & 0 & is & is & 3 & z & is \\
\hline Slope in degrees (Nagib v stopinjah) & & 20 & 70 & 45 & 40 & 45 & 20 & 5 & 0 & 5 & 40 & 35 & 40 & 15 \\
\hline Parent material (Matična podlaga) & & $\mathrm{A}$ & A & A & A & A & A & A & A & $\mathrm{A}$ & A & A & A & A \\
\hline Soil (Tla) & & $\mathrm{Li}$ & $\mathrm{Li}$ & $\mathrm{R}$ & $\mathrm{Li}$ & $\mathrm{Li}$ & $\mathrm{R}$ & $\mathrm{R}$ & $\mathrm{R}$ & $\mathrm{R}$ & $\mathrm{R}$ & $\mathrm{R}$ & $\mathrm{R}$ & $\mathrm{R}$ \\
\hline Stoniness in \% (Kamnitost $\mathrm{v} \%)$ & & 30 & 50 & 60 & 30 & 20 & 30 & 20 & 0 & 0 & 30 & 10 & 30 & 50 \\
\hline Cover in \% (Zastiranje v \%): & & & & & & & & & & & & & & \\
\hline Tree layer (Drevesna plast) & E3 & . & . & . & . & . & . & . & . & . & 80 & 70 & 80 & 70 \\
\hline Shrub layer (Grmovna plast) & E2 & . & . & 20 & . & 5 & 40 & 20 & 5 & 10 & 40 & 20 & 50 & 40 \\
\hline Herb layer (Zeliščna plast) & E1 & 70 & 50 & 70 & 80 & 90 & 70 & 80 & 95 & 100 & 80 & 90 & 70 & 60 \\
\hline Moss layer (Mahovna plast) & E0 & . & . & . & . & . & 10 & . & . & . & 5 & 5 & 10 & 10 \\
\hline Maximum diameter (Največji prsni premer) & $\mathrm{cm}$ & . & . & . & . & . & . & . & . & . & 20 & 15 & 20 & 25 \\
\hline Maximum height (Največja drevesna višina) & $\mathrm{m}$ & . & . & . & . & . & . & . & . & . & 10 & 8 & 10 & 18 \\
\hline Relevé area (Velikost popisne ploskve) & $\mathrm{m}^{2}$ & 10 & 10 & 10 & 15 & 10 & 10 & 10 & 10 & 20 & 100 & 100 & 100 & 200 \\
\hline Number of species (Število vrst) & & 29 & 28 & 47 & 49 & 57 & 45 & 38 & 51 & 55 & 61 & 55 & 77 & 79 \\
\hline Date of taking relevé (Datum popisa) & & 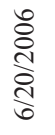 & 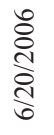 & 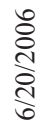 & 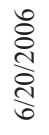 & 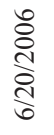 & 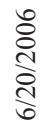 & 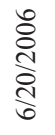 & 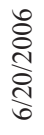 & 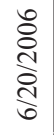 & 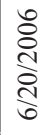 & 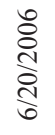 & 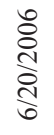 & $\begin{array}{l}\stackrel{0}{\circ} \\
\stackrel{N}{ } \\
\stackrel{\overbrace{}}{0}\end{array}$ \\
\hline Locality (Nahajališče) & & 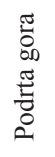 & 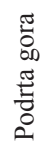 & 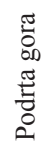 & 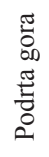 & 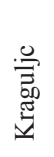 & 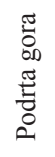 & 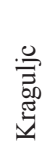 & 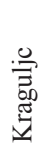 & $\begin{array}{l}\overrightarrow{\overrightarrow{0}} \\
\text { ov } \\
\overrightarrow{0} \\
\overrightarrow{0} \\
\overrightarrow{0}\end{array}$ & 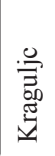 & 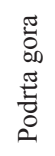 & 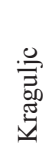 & 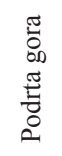 \\
\hline Quadrant (Kvadrant) & & $\frac{\stackrel{N}{a}}{\stackrel{a}{\sigma}}$ & $\frac{\mathfrak{d}}{a}$ & 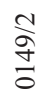 & 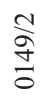 & $\frac{\stackrel{N}{a}}{\stackrel{a}{\sigma}}$ & $\frac{\stackrel{N}{a}}{\stackrel{a}{\sigma}}$ & $\frac{\mathfrak{d}}{\stackrel{a}{\sigma}}$ & $\frac{\stackrel{N}{a}}{\sigma}$ & $\frac{\stackrel{N}{a}}{\sigma}$ & $\frac{\stackrel{N}{a}}{\stackrel{d}{\sigma}}$ & $\frac{d}{a}$ & 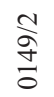 & 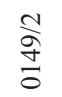 \\
\hline
\end{tabular}

Differential species of the ass. Genisto sericeae-Seslerietum kalnikensis

AT Sempervivum tectorum

AT Athamanta turbith

Differential species of successional stages

TG Laserpitium siler

Differential species of the ass. Seslerio autumnalis-Ostryetum

QP Sesleria autumnalis

QP Sorbus aria

QP Sorbus aria

SS Satureion subspicatae

Sesleria juncifolia subsp. kalnikensis Artemisia alba

Satureja montana subsp. variegata

Genista sericea
Pr. Fr. Pr. Fr.

$$
\text { . }
$$




\begin{tabular}{|c|c|c|c|c|c|c|c|c|c|c|c|c|c|c|c|c|c|c|c|c|}
\hline \multirow{2}{*}{\multicolumn{2}{|c|}{ Number of relevé (Številka popisa) }} & & \multicolumn{9}{|c|}{ I } & \multicolumn{4}{|c|}{ II } & \multicolumn{3}{|c|}{$\mathrm{I}$} & \multicolumn{2}{|l|}{ II } \\
\hline & & & 1 & 2 & 3 & 4 & 5 & 6 & 7 & 8 & 9 & 10 & 11 & 12 & 13 & Pr. & Fr. & Pr. & Fr. & \\
\hline & Dianthus tergestinus & E1 & + & + & + & . & + & . & + & + & . & . & . & . & . & 6 & 67 & 0 & 0 & 6 \\
\hline & Satureja subspicata subsp. liburnica & E1 & . & + & . & . & + & . & . & 1 & + & . & . & . & . & 4 & 44 & 0 & 0 & 4 \\
\hline & Iris pallida subsp. illyrica & E1 & . & . & . & 2 & . & . & . & . & + & . & + & . & . & 2 & 22 & 1 & 25 & 3 \\
\hline & Gentiana verna subsp. tergestina & E1 & . & . & . & . & . & . & . & $\mathrm{r}$ & . & . & . & . & . & 1 & 11 & 0 & 0 & 1 \\
\hline \multirow{5}{*}{ SV } & Scorzoneretalia villosae & & & & & & & & & & & & & & & & & & & \\
\hline & Pseudolysimachion barrelieri & E1 & . & + & + & + & 1 & + & . & + & + & + & . & + & . & 7 & 78 & 2 & 50 & 9 \\
\hline & Potentilla tommasiniana & E1 & . & . & + & 1 & + & + & + & + & . & . & . & . & . & 6 & 67 & 0 & 0 & 6 \\
\hline & Sanguisorba muricata & E1 & . & . & + & 1 & + & . & + & + & + & . & . & . & . & 6 & 67 & 0 & 0 & 6 \\
\hline & Potentilla australis & E1 & . & . & . & . & . & . & . & + & . & . & . & . & . & 1 & 11 & 0 & 0 & 1 \\
\hline \multirow[t]{2}{*}{ FB } & Festuco-Brometea & & & & & & & & & & & & & & & & & & & \\
\hline & Allium carinatum subsp. pulchellum & E1 & + & + & + & + & + & . & + & 1 & 1 & + & 1 & . & + & 8 & 89 & 3 & 75 & 11 \\
\hline & Bromus erectus agg. & E1 & + & + & 1 & + & 1 & + & 1 & + & + & + & . & + & . & 9 & 100 & 2 & 50 & 11 \\
\hline & Galium lucidum & E1 & . & + & + & + & 1 & 2 & 1 & + & + & + & . & + & . & 8 & 89 & 2 & 50 & 10 \\
\hline & Dianthus monspessulanus & E1 & + & . & + & + & + & + & . & + & + & + & . & + & . & 7 & 78 & 2 & 50 & 9 \\
\hline & Carex humilis & E1 & 1 & + & 1 & . & 1 & 2 & . & 1 & + & . & . & + & . & 7 & 78 & 1 & 25 & 8 \\
\hline & Centaurea triumfettii & E1 & 1 & + & + & 1 & 1 & 1 & . & + & + & . & . & . & . & 8 & 89 & 0 & 0 & 8 \\
\hline & Euphorbia cyparissias & E1 & . & . & + & + & + & 1 & + & + & + & . & + & . & . & 7 & 78 & 1 & 25 & 8 \\
\hline & Teucrium montanum & E1 & + & + & + & + & + & + & . & . & + & . & . & . & . & 7 & 78 & 0 & 0 & 7 \\
\hline & Anthyllis vulneraria & E1 & . & . & + & + & + & + & + & . & + & . & + & . & . & 6 & 67 & 1 & 25 & 7 \\
\hline & Melica ciliata & E1 & + & + & 1 & + & + & . & . & . & . & + & . & . & . & 5 & 56 & 1 & 25 & 6 \\
\hline & Globularia cordifolia & E1 & + & . & . & + & + & . & + & + & . & . & . & . & . & 5 & 56 & 0 & 0 & 5 \\
\hline & Festuca rupicola & E1 & . & . & . & + & + & + & 1 & 1 & . & . & . & . & . & 5 & 56 & 0 & 0 & 5 \\
\hline & Peucedanum oreoselinum & E1 & . & . & . & . & + & + & . & . & . & . & + & . & + & 2 & 22 & 2 & 50 & 4 \\
\hline & Poa angustifolia & E1 & . & . & . & . & . & + & . & . & . & 1 & 2 & + & . & 1 & 11 & 3 & 75 & 4 \\
\hline & Teucrium chamaedrys & E1 & . & . & + & 1 & . & . & . & + & . & . & . & . & + & 3 & 33 & 1 & 25 & 4 \\
\hline & Thymus pulegioides & E1 & . & . & . & . & + & . & + & + & 1 & . & . & . & . & 4 & 44 & 0 & 0 & 4 \\
\hline & Orobanche gracilis & E1 & + & . & . & + & . & . & . & . & + & . & . & . & . & 3 & 33 & 0 & 0 & 3 \\
\hline & Allium senescens & E1 & . & . & + & + & . & . & . & + & . & . & . & . & . & 3 & 33 & 0 & 0 & 3 \\
\hline & Ajuga genevensis & E1 & . & . & . & + & . & . & . & + & . & . & . & . & + & 2 & 22 & 1 & 25 & 3 \\
\hline & Helianthemum ovatum & E1 & . & . & . & + & . & + & 1 & . & . & . & . & . & . & 3 & 33 & 0 & 0 & 3 \\
\hline & Plantago media (inc. var. urvilleana) & E1 & . & . & . & . & . & . & + & + & + & . & . & . & . & 3 & 33 & 0 & 0 & 3 \\
\hline & Dorycnium germanicum & E1 & . & . & . & . & + & . & . & 1 & . & . & . & . & . & 2 & 22 & 0 & 0 & 2 \\
\hline & Trifolium montanum & E1 & . & . & . & . & . & . & 1 & 1 & . & . & . & . & . & 2 & 22 & 0 & 0 & 2 \\
\hline & Scabiosa triandra & E1 & . & . & . & . & . & . & . & + & + & . & . & . & . & 2 & 22 & 0 & 0 & 2 \\
\hline & Koeleria pyramidata & E1 & . & . & . & . & . & . & . & + & + & . & . & . & . & 2 & 22 & 0 & 0 & 2 \\
\hline & Centaurea pannonica & E1 & . & . & . & . & . & . & . & + & + & . & . & . & . & 2 & 22 & 0 & 0 & 2 \\
\hline & Galium purpureum & E1 & . & . & . & + & . & . & . & . & . & . & . & . & . & 1 & 11 & 0 & 0 & 1 \\
\hline & Stachys recta & E1 & . & . & . & . & + & . & . & . & . & . & . & . & . & 1 & 11 & 0 & 0 & 1 \\
\hline & Thlaspi praecox & E1 & . & . & . & . & . & . & . & + & . & . & . & . & . & 1 & 11 & 0 & 0 & 1 \\
\hline & Hippocrepis comosa & E1 & . & . & . & . & . & . & . & . & 1 & . & . & . & . & 1 & 11 & 0 & 0 & 1 \\
\hline & Gymnadenia conopsea & E1 & . & . & . & . & . & . & . & . & + & . & . & . & . & 1 & 11 & 0 & 0 & 1 \\
\hline & Pimpinella saxifraga & E1 & . & . & . & . & . & . & . & . & + & . & . & . & . & 1 & 11 & 0 & 0 & 1 \\
\hline & Briza media & E1 & . & . & . & . & . & . & . & . & + & . & . & . & . & 1 & 11 & 0 & 0 & 1 \\
\hline & Festuca valesiaca & E1 & . & . & . & . & . & . & . & . & + & . & . & . & . & 1 & 11 & 0 & 0 & 1 \\
\hline & Brachypodium rupestre & E1 & . & . & . & . & . & . & . & . & . & 1 & . & . & . & 0 & 0 & 1 & 25 & 1 \\
\hline & Carlina vulgaris & E1 & . & . & . & . & . & . & . & . & . & + & . & . & . & 0 & 0 & 1 & 25 & 1 \\
\hline & Medicago lupulina & E1 & . & . & . & . & . & . & . & . & . & . & + & . & . & 0 & 0 & 1 & 25 & 1 \\
\hline & Arabis hirsuta & E1 & . & . & . & . & . & . & . & . & . & . & . & + & . & 0 & 0 & 1 & 25 & 1 \\
\hline & Allium carinatum subsp. carinatum & E1 & . & . & . & . & . & . & . & . & . & . & . & . & + & 0 & 0 & 1 & 25 & 1 \\
\hline
\end{tabular}




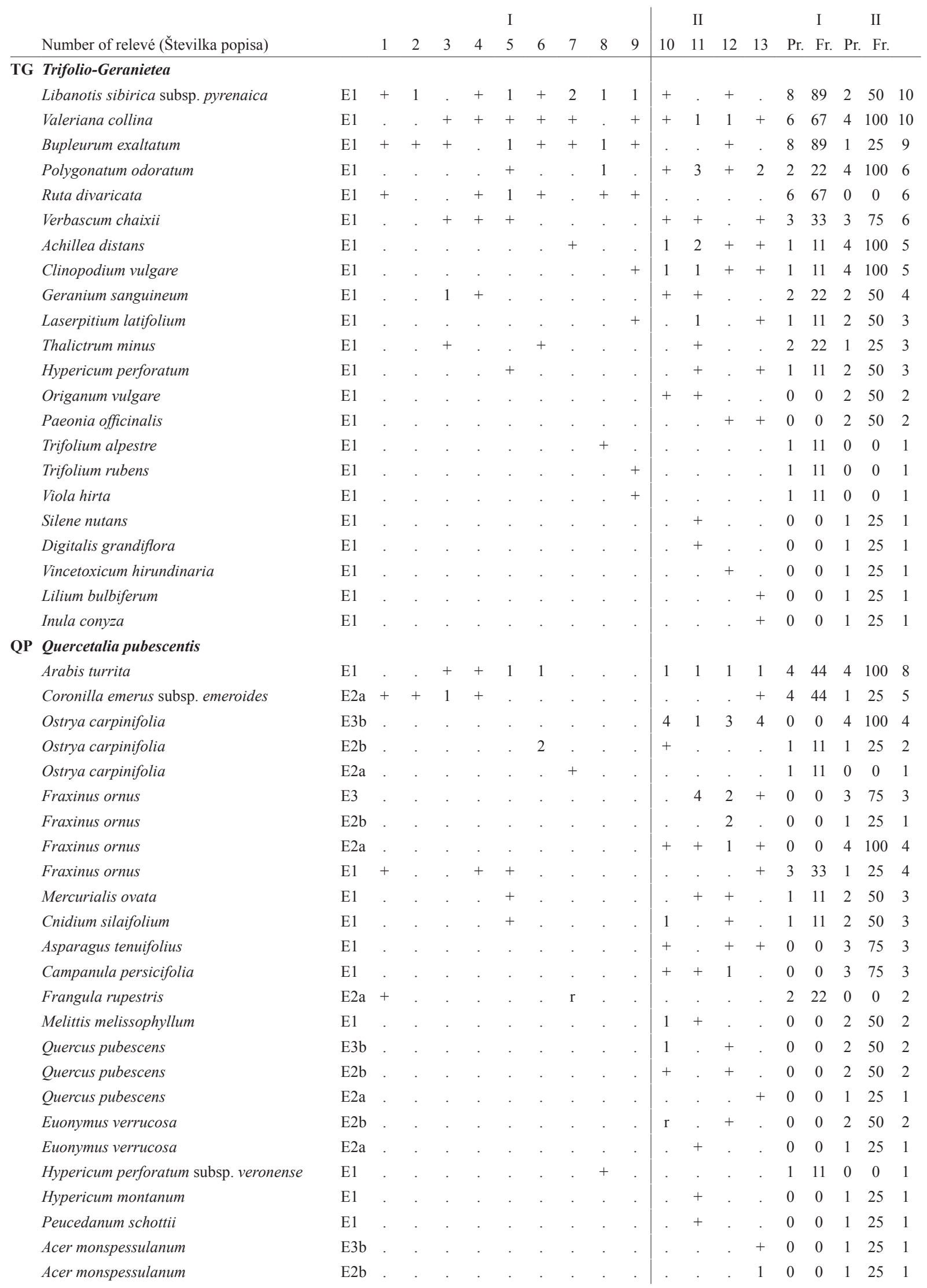




\begin{tabular}{|c|c|c|c|c|c|c|c|c|c|c|c|c|c|c|c|c|c|c|c|c|}
\hline & & & & & & & I & & & & & & II & & & & I & & II & \\
\hline & Number of relevé (Številka popisa) & & 1 & 2 & 3 & 4 & 5 & 6 & 7 & 8 & 9 & 10 & 11 & 12 & 13 & Pr. & Fr. & Pr. & Fr. & \\
\hline & Acer monspessulanum & E2a & . & . & . & . & . & . & . & . & . & . & . & . & 1 & 0 & 0 & 1 & 25 & 1 \\
\hline & Cornus mas & E2a & . & . & . & . & . & . & . & . & . & . & . & . & + & 0 & 0 & 1 & 25 & 1 \\
\hline \multirow[t]{28}{*}{$\mathbf{F}$} & Fagetalia sylvaticae & & & & & & & & & & & & & & & & & & & \\
\hline & Geranium robertianum & E1 & . & . & . & . & . & . & . & . & . & + & + & + & + & 0 & 0 & 4 & 100 & 4 \\
\hline & Acer platanoides & E1 & . & . & . & . & + & . & . & . & . & . & . & 1 & . & 1 & 11 & 1 & 25 & 2 \\
\hline & Asarum europaeum subsp. caucasicum & E1 & . & . & . & . & . & . & . & . & . & . & . & 1 & 1 & 0 & 0 & 2 & 50 & 2 \\
\hline & Salvia glutinosa & E1 & . & . & . & . & . & . & . & . & . & . & . & + & + & 0 & 0 & 2 & 50 & 2 \\
\hline & Campanula trachelium & E1 & . & . & . & . & . & . & . & . & . & . & . & + & 1 & 0 & 0 & 2 & 50 & 2 \\
\hline & Fagus sylvatica & $\mathrm{E} 3 \mathrm{~b}$ & . & . & . & . & . & . & . & . & . & . & . & + & 1 & 0 & 0 & 2 & 50 & 2 \\
\hline & Fagus sylvatica & $\mathrm{E} 2 \mathrm{~b}$ & . & . & . & . & . & . & . & . & . & . & . & . & + & 0 & 0 & 1 & 25 & 1 \\
\hline & Fagus sylvatica & $\mathrm{E} 2 \mathrm{a}$ & . & . & . & . & . & + & . & . & . & . & . & . & . & 1 & 11 & 0 & 0 & 1 \\
\hline & Acer pseudoplatanus & E3a & . & . & . & . & . & . & . & . & . & . & . & . & + & 0 & 0 & 1 & 25 & 1 \\
\hline & Acer pseudoplatanus & $\mathrm{E} 2 \mathrm{a}$ & . & . & . & . & . & . & . & . & . & . & . & + & . & 0 & 0 & 1 & 25 & 1 \\
\hline & Acer pseudoplatanus & E1 & . & . & . & . & . & . & . & . & + & . & . & . & . & 1 & 11 & 0 & 0 & 1 \\
\hline & Mercurialis perennis & E1 & . & . & . & . & . & . & . & . & . & + & . & . & . & 0 & 0 & 1 & 25 & 1 \\
\hline & Poa nemoralis & E1 & . & . & . & . & . & . & . & . & . & + & . & . & . & 0 & 0 & 1 & 25 & 1 \\
\hline & Prunus avium & E1 & . & . & . & . & . & . & . & . & . & + & . & . & . & 0 & 0 & 1 & 25 & 1 \\
\hline & Corydalis solida & E1 & . & . & . & . & . & . & . & . & . & $\mathrm{r}$ & . & . & . & 0 & 0 & 1 & 25 & 1 \\
\hline & Fraxinus excelsior & $\mathrm{E} 2 \mathrm{~b}$ & . & . & . & . & . & . & . & . & . & $\mathrm{r}$ & . & . & . & 0 & 0 & 1 & 25 & 1 \\
\hline & Dryopteris filix-mas & E1 & . & . & . & . & . & . & . & . & . & . & . & + & . & 0 & 0 & 1 & 25 & 1 \\
\hline & Polystichum aculeatum & E1 & . & . & . & . & . & . & . & . & . & . & . & + & . & 0 & 0 & 1 & 25 & 1 \\
\hline & Aruncus dioicus & E1 & . & . & . & . & . & . & . & . & . & . & . & 1 & . & 0 & 0 & 1 & 25 & 1 \\
\hline & Cyclamen purpurascens & E1 & . & . & . & . & . & . & . & . & . & . & . & + & . & 0 & 0 & 1 & 25 & 1 \\
\hline & Daphne mezereum & E2a & . & . & . & . & . & . & . & . & . & . & . & + & . & 0 & 0 & 1 & 25 & 1 \\
\hline & Thalictrum aquilegiifolium & E1 & . & . & . & . & . & . & . & . & . & . & . & + & . & 0 & 0 & 1 & 25 & 1 \\
\hline & Calamintha grandiflora & E1 & . & . & . & . & . & . & . & . & . & . & . & . & + & 0 & 0 & 1 & 25 & 1 \\
\hline & Mycelis muralis & E1 & . & . & . & . & . & . & . & . & . & . & . & . & + & 0 & 0 & 1 & 25 & 1 \\
\hline & Epipactis helleborine & E1 & . & . & . & . & . & . & . & . & . & . & . & . & + & 0 & 0 & 1 & 25 & 1 \\
\hline & Rhamnus fallax & $\mathrm{E} 2 \mathrm{a}$ & . & . & . & . & . & . & . & . & . & . & . & . & + & 0 & 0 & 1 & 25 & 1 \\
\hline & Tilia platyphyllos & $\mathrm{E} 3 \mathrm{~b}$ & . & . & . & . & . & . & . & . & . & . & . & . & $\mathrm{r}$ & 0 & 0 & 1 & 25 & 1 \\
\hline \multirow{14}{*}{ QF } & Querco-Fagetea & & & & & & & & & & & & & & & & & & & \\
\hline & Galanthus nivalis & E1 & . & . & . & + & . & 1 & . & . & . & 1 & 1 & 1 & 1 & 2 & 22 & 4 & 100 & 6 \\
\hline & Chamaecytisus supinus & E1 & . & . & + & + & . & . & . & . & + & . & + & . & . & 3 & 33 & 1 & 25 & 4 \\
\hline & Hepatica nobilis & E1 & . & . & . & . & + & . & . & . & . & . & 1 & 1 & + & 1 & 11 & 3 & 75 & 4 \\
\hline & Cruciata glabra & E1 & . & . & . & . & . & . & . & + & 1 & . & . & . & . & 2 & 22 & 0 & 0 & 2 \\
\hline & Carex digitata & E1 & . & . & . & . & . & . & . & . & + & . & . & + & . & 1 & 11 & 1 & 25 & 2 \\
\hline & Viola riviniana & E1 & . & . & . & . & . & . & . & . & . & + & . & + & . & 0 & 0 & 2 & 50 & 2 \\
\hline & Anemone nemorosa & E1 & . & . & . & . & . & . & . & . & . & . & 1 & 1 & . & 0 & 0 & 2 & 50 & 2 \\
\hline & Corylus avellana & $\mathrm{E} 2 \mathrm{~b}$ & . & . & . & . & . & . & . & . & . & . & . & + & 1 & 0 & 0 & 2 & 50 & 2 \\
\hline & Primula vulgaris & E1 & . & . & . & . & . & . & . & . & . & . & + & . & . & 0 & 0 & 1 & 25 & 1 \\
\hline & Hypericum hirsutum & E1 & . & . & . & . & . & . & . & . & . & . & . & 1 & . & 0 & 0 & 1 & 25 & 1 \\
\hline & Taxus baccata & $\mathrm{E} 2 \mathrm{a}$ & . & . & . & . & . & . & . & . & . & . & . & $\mathrm{r}$ & . & 0 & 0 & 1 & 25 & 1 \\
\hline & Geum urbanum & E1 & . & . & . & . & . & . & . & . & . & . & . & . & + & 0 & 0 & 1 & 25 & 1 \\
\hline & Solidago virgaurea & E1 & . & . & . & . & . & . & . & . & . & . & . & . & + & 0 & 0 & 1 & 25 & 1 \\
\hline \multirow[t]{5}{*}{$\mathbf{R P}$} & Rhamno-Prunetea s. lat. & & & & & & & & & & & & & & & & & & & \\
\hline & Prunus mahaleb & $\mathrm{E} 2 \mathrm{~b}$ & . & . & . & . & . & . & . & . & . & . & . & . & + & 0 & 0 & 1 & 25 & 1 \\
\hline & Prunus mahaleb & $\mathrm{E} 2 \mathrm{a}$ & . & . & . & . & . & + & + & . & + & . & . & + & . & 3 & 33 & 1 & 25 & 4 \\
\hline & Euonymus europaea & $\mathrm{E} 2 \mathrm{~b}$ & . & . & . & . & . & . & . & . & . & 1 & . & . & . & 0 & 0 & 1 & 25 & 1 \\
\hline & Euonymus europaea & $\mathrm{E} 2 \mathrm{a}$ & . & . & . & . & . & . & . & . & + & 2 & . & + & 1 & 1 & 11 & 3 & 75 & 4 \\
\hline
\end{tabular}




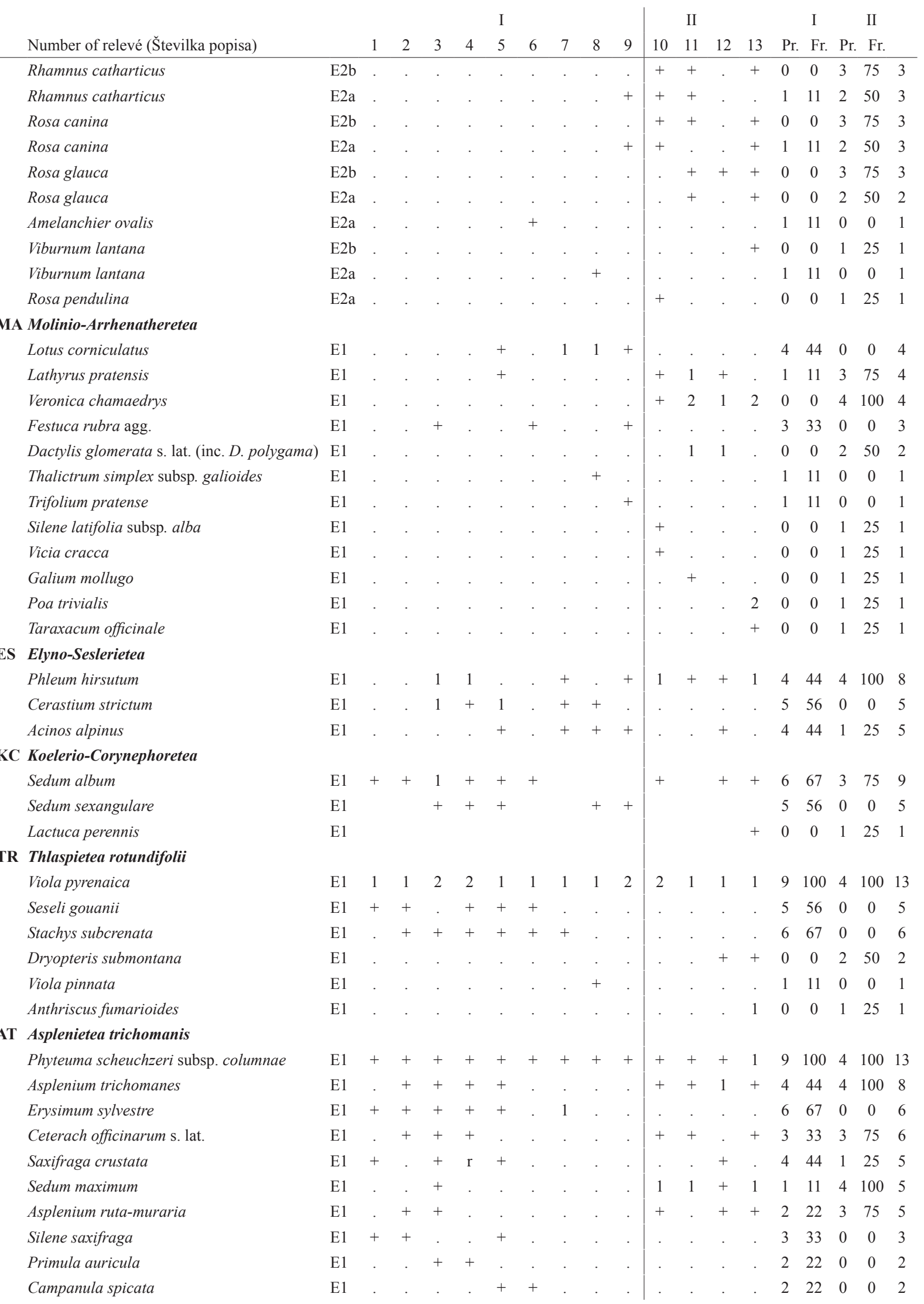




\begin{tabular}{|c|c|c|c|c|c|c|c|c|c|c|c|c|c|c|c|c|c|c|c|c|}
\hline & & & & & & & I & & & & & & II & & & & I & & II & \\
\hline & Number of relevé (Številka popisa) & & 1 & 2 & 3 & 4 & 5 & 6 & 7 & 8 & 9 & 10 & 11 & 12 & 13 & Pr. & Fr. & Pr. & Fr. & \\
\hline & Moehringia muscosa & E1 & . & . & . & . & . & . & . & . & . & . & . & + & + & 0 & 0 & 2 & 50 & 2 \\
\hline & Polypodium vulgare & E1 & . & . & . & . & . & . & . & . & . & . & . & + & + & 0 & 0 & 2 & 50 & 2 \\
\hline & Micromeria thymifolia & E1 & . & . & . & . & . & . & . & . & . & + & . & . & . & 0 & 0 & 1 & 25 & 1 \\
\hline & Cystopteris fragilis & E1 & . & . & . & . & . & . & . & . & . & . & . & + & . & 0 & 0 & 1 & 25 & 1 \\
\hline & Valeriana tripteris & E1 & . & . & . & . & . & . & . & . & . & . & . & + & . & 0 & 0 & 1 & 25 & 1 \\
\hline & Sedum hispanicum & E1 & . & . & . & . & . & . & . & . & . & . & . & . & + & 0 & 0 & 1 & 25 & 1 \\
\hline & Campanula pyramidalis & E1 & . & . & . & . & . & . & . & . & . & . & . & . & + & 0 & 0 & 1 & 25 & 1 \\
\hline $\mathbf{O}$ & Other species (Druge vrste) & & & & & & & & & & & & & & & & & & & \\
\hline & Fragaria vesca & E1 & . & . & . & . & . & + & + & . & . & + & + & . & 1 & 2 & 22 & 3 & 75 & 5 \\
\hline & Rubus idaeus & $\mathrm{E} 2 \mathrm{a}$ & . & . & . & . & + & . & + & . & . & . & . & + & . & 2 & 22 & 1 & 25 & 3 \\
\hline & Juniperus communis & $\mathrm{E} 2 \mathrm{~b}$ & . & . & . & . & . & + & . & . & . & . & . & . & . & 1 & 11 & 0 & 0 & 1 \\
\hline & Juniperus communis & $\mathrm{E} 2 \mathrm{a}$ & . & . & . & . & . & + & . & + & 1 & . & . & . & . & 3 & 33 & 0 & 0 & 3 \\
\hline & Potentilla erecta & E1 & . & . & . & . & . & . & . & + & + & . & . & . & . & 2 & 22 & 0 & 0 & 2 \\
\hline & Fallopia dumetorum & E1 & . & . & . & . & . & . & . & . & . & + & + & . & . & 0 & 0 & 2 & 50 & 2 \\
\hline & Lamium maculatum & E1 & . & . & . & . & . & . & . & . & . & 1 & . & . & 1 & 0 & 0 & 2 & 50 & 2 \\
\hline & Rubus caesius & $\mathrm{E} 2 \mathrm{a}$ & . & . & . & . & . & . & . & . & . & + & . & . & . & 0 & 0 & 1 & 25 & 1 \\
\hline $\mathbf{M l}$ & Mosses and lichens (Mahovi in liša & & & & & & & & & & & & & & & & & & & \\
\hline & Homalothecium lutescens & E0 & . & . & . & . & . & 1 & 1 & + & . & + & . & . & 1 & 3 & 33 & 2 & 50 & 5 \\
\hline & Homalothecium sericeum & E0 & . & . & + & . & + & 1 & . & . & . & . & . & + & . & 3 & 33 & 1 & 25 & 4 \\
\hline & Ctenidium molluscum & E0 & . & . & . & . & . & 2 & . & . & . & + & . & 1 & . & 1 & 11 & 2 & 50 & 3 \\
\hline & Isothecium alopecuroides & E0 & . & . & . & . & . & + & . & . & . & . & + & . & + & 1 & 11 & 2 & 50 & 3 \\
\hline & Porella platyphylla & E0 & . & . & + & . & . & . & . & . & . & . & . & . & + & 1 & 11 & 1 & 25 & 2 \\
\hline & Hypnum cupressiforme & E0 & . & . & . & . & + & + & . & . & . & . & . & . & . & 2 & 22 & 0 & 0 & 2 \\
\hline & Homalothecium philippeanum & E0 & . & . & . & . & . & . & . & . & . & . & + & . & + & 0 & 0 & 2 & 50 & 2 \\
\hline & Marchantia polymorpha & E0 & . & . & . & . & . & . & . & . & . & . & + & + & . & 0 & 0 & 2 & 50 & 2 \\
\hline & Tortella tortuosa & E0 & . & . & . & . & . & . & . & . & . & . & . & + & + & 0 & 0 & 2 & 50 & 2 \\
\hline & Polytrichum formosum & E0 & . & . & . & . & . & . & . & . & . & + & . & . & . & 0 & 0 & 1 & 25 & 1 \\
\hline & Neckera crispa & E0 & . & . & . & . & . & . & . & . & . & . & . & + & . & 0 & 0 & 1 & 25 & 1 \\
\hline & Rhytidiadelphus triquetrus & E0 & . & . & . & . & . & . & . & . & . & . & . & + & . & 0 & 0 & 1 & 25 & 1 \\
\hline & Anomodon viticulosus & E0 & . & . & . & . & . & . & . & . & . & . & . & . & + & 0 & 0 & 1 & 25 & 1 \\
\hline & Plagiomnium cuspidatum & E0 & . & . & . & . & . & . & . & . & . & . & . & . & + & 0 & 0 & 1 & 25 & 1 \\
\hline & Schistidium apocarpum & E0 & . & . & . & . & . & . & . & . & . & . & . & . & + & 0 & 0 & 1 & 25 & 1 \\
\hline & Peltigera canina & E0 & . & . & . & . & . & . & . & . & . & . & . & . & + & 0 & 0 & 1 & 25 & 1 \\
\hline
\end{tabular}

